\title{
SUR, A Program to Generate Error Covariance Files
}

\author{
F. C. Difilippo \\ A ROTICE
}

PORTIONS OF THIS REPORT ARE ILLEGIBLE. It has been reproduced from the best available copy to permit the broadest possible availability.

\section{OAK RIDGE NATIONAL LABORATORY}




\section{DISCLAIMER}

This report was prepared as an account of work sponsored by an agency of the United States Government. Neither the United States Government nor any agency Thereof, nor any of their employees, makes any warranty, express or implied, or assumes any legal liability or responsibility for the accuracy, completeness, or usefulness of any information, apparatus, product, or process disclosed, or represents that its use would not infringe privately owned rights. Reference herein to any specific commercial product, process, or service by trade name, trademark, manufacturer, or otherwise does not necessarily constitute or imply its endorsement, recommendation, or favoring by the United States Government or any agency thereof. The views and opinions of authors expressed herein do not necessarily state or reflect those of the United States Government or any agency thereof. 


\section{DISCLAIMER}

Portions of this document may be illegible in electronic image products. Images are produced from the best available original document. 
Printed in the United States of America. Available from National Technical Information Service

U.S. Department of Commerce

5285 Port Royal Road, Springfield, Virginia 22161

Price: Printed Copy \$4.50; Microfiche \$2.25

This report was prepared as an account of work sponsored by the United States Government. Neither the United States nor the Energy Research and Development Administration, nor any of their employees, nor any of their contractors, subcontractors, or their employees, makes any warranty, express or implied, or assumes any legal liability or responsibility for the accuracy, completeness or usefulness of any information, apparatus, product or process disclosed, or represents that its use would not infringe privately owned rights. 
Contract No. W-7405-eng-26

Neutron Physics Division

SUR, A PROGRAM TO GENERATE ERROR COVARIANCE FILES

F. C. Difilippo*

* On assignment from Comisión Nacional de Energía Atómica, Argentina.

\section{MARCH 1976}

NOTICE This documant contains information of a preliminary nature and was prepared primerlly for Internal use at the Oak Ridge National Laboratory. It is subject to revision or correction and therefore does not represent a final report.

OAK RIDGE NATIONAL LABORATORY

Oak Ridge, Tennessee 37830

operated by

UNION CARBIDE CORPORATION

for the

ENERGY RESEARCH AND DEVELOPMENT ADMTINISTRAT,ION 
THIS PAGE

\section{WAS INTENTIONALLY LEFT BLANK}


Acknowledgments • • . . . . . . . . . . . . . . . . . . . . . v

Abstract . . . . . . . . . . . . . . . . . . . . . . . . vii

I. Introduction . . . . . . . . . . . . . . . . . . . . . . 1

II. Calculation of Relative Covariance Matrices . . . . . . . . 1

III. Brief Description of the Program . . . . . . . . . . . . . . . 2

IV. Description of the Input-Output Specifications . . . . . . . . 4

V. Listing of the Program and Sample Problem . . . . . . . . . . 8

VI. A Three-Dimensional Correlation Matrix Plot . • • • • • • • . 21 References . • • • • • • • • • • • • • • • • • • • • • • 23

Appendix A. List of References Used in the Calculation of the Covariance Matrices . . . . . . . . . . . . . . . . 25

Appendix B. Standard Deviation and Correlation Matrices Corresponding to Different Cross Sections . . . . . . . . . . . 
THIS PAGE

\section{WAS INTENTIONALLY \\ LEFT BLANK}




\section{Acknowledgments}

The author is indebted to R. Gwin and L. W. Weston for providing the literature search for the plutonium isotopes cross sections. F. G. Perey, C. R. Weisbin, G, de Saussure, R. W. Peelle, and R. B. Perez have participated in many discussions regarding the present work. Thanks are also due J. D. Drischler for the correlation matrix plot. The convenient output presented in Appendix B was programmed by R. W. Peelle. 
THIS PAGE

WAS INTENTIONALLY LEFT BLANK 


\section{Abstract}

Covariance matrices were calculated for the ${ }^{238} \mathrm{U}, 24{ }^{24} \mathrm{Pu}$, and ${ }^{239} \mathrm{Pu}$

fission cross sections and for the ${ }^{238} \mathrm{U},{ }^{240} \mathrm{Pu},{ }^{241} \mathrm{Pu}$, and ${ }^{239} \mathrm{Pu}$ capture cross sections. A computer program was written which uses the evaluated ENDF/B data files and the measured or evaluated (from other evaluations) cross sections for the calculation of the uncertainty files. An effort has been made to make the output of the program consistent with the ENDF/B error files format. A user's manual for the present code and references utilized in the covariance matrix calculations are given. 


\section{INTRODUCTION}

The economic impact of nuclear data uncertainties in fast breeder as well as in thermal reactors has been the subject of many studies. $1-5$ To evaluate the effect of group cross-section uncertainties in sensitivity calculations, the correlation both within the group structure and among different isotopes has to be calculated. Extensive discussions of this procedure are given in refs, 6-8.

\section{CALCULATION OF RELATIVE COVARIANCE MATRICES}

The relative covariance matrix elements are defined here as:

$$
\operatorname{cov}(I, J)=\left\langle\left(\frac{x_{i}^{l}-x_{i}}{x_{i}}\right)\left(\frac{x_{j}^{l}-x_{j}}{x_{j}}\right)\right\rangle,
$$

where $x_{i}^{\ell}$ and $x_{j}^{\ell}$ are the group average cross sections for the $i^{\text {th }}$ and $j^{\text {th }}$ energy groups, respectively, for the $e^{\text {th }}$ data set. The cross sections $x_{i}$ and $x_{j}$ are the reference ("true") values, which by convention are taken to be the corresponding ENDF/B values. The bracket denotes a weighted average over the ensemble of data sets, having common data in the $i^{\text {th }}$ and $j^{\text {th }}$ groups, where weights, $W_{\ell}$, have been assigned to each data set.

The. ENDF/B-IV files, used as the reference data, were energy-weighted by a flat weighting function in producing the group-average cross sections. Details of calculations are given in Sect. III. 
The present study of the error covariance matrix implies an "external" analysis of the data. Each data set, whether the results of a measurement or of an evaluation, is assigned an arbitrary weight and is compared with a reference line taken to be the ENDF/B-IV evaluation.

\section{BRIEF DESCRIPTION OF THE PROGRAM}

The calculations were done by the code SUR, which, in its present form, only handles error correlation for a given cross-section data set within a given energy range. A description of the main steps in the program follows.

Step 1: The program sets the energy limits of the group structure for several options: (1) constant lethargy interval, (2) decimal interval, or (3) a mixture of the first two.

Slep 2: For the ratio measurements, the standard reference cross section was always the ENDF/B-IV evaluation.

Step 3: The measurements corresponding to the data set $\ell$, inside a group $j$, are reduced to the mean energy of the group. This is implemented by approximating the shape of the cross section with a parabola determincd by the evaluated cross sections in groups $j-1, j, j+1$. Then, all the measurements corresponding to the data set $\ell$, inside group $j$, are averaged. 
Step 4: Whenever there are not measurements in the $\ell^{\text {th }}$ set: of data, inside group $j$; the program makes a linear interpolation of data set, $\ell$, values corresponding to the neighboring groups. Before implementing the interpolation, the program searches whether or not the data points for the interpolation procedure are within energy limits preset by the user. If not, the interpolation is not made.

Step 5: Calculation of $\operatorname{COV}(I, J)$ is made. This is done by the formula

$$
\operatorname{cov}(I, J)=\frac{1}{R R} \sum_{K=1}^{N A}\left[\frac{\sigma(K, I)-\sigma(I)}{\sigma(I)}\right]:\left[\frac{\sigma\left(K_{2}, J\right)-\sigma(J)}{\sigma(J)}\right] W(K),
$$

where

$$
\begin{aligned}
\mathrm{RR}=\sum_{\mathrm{K}=1}^{\mathrm{NA}} \mathrm{W}(\mathrm{K}), & \\
\sigma(\mathrm{K}, \mathrm{I}), \sigma(\mathrm{K}, \mathrm{J})= & \text { measured or interpolated cross sections of data } \\
& \text { set } \mathrm{K} \text {, in groups } \mathrm{I} \text { and } \mathrm{J}, \\
\sigma(\mathrm{I}), \sigma(\mathrm{J})= & \text { evaluated cross sections of groups } \mathrm{I} \text { and } \mathrm{J}, \\
\mathrm{W}(\mathrm{K})= & \text { weight of data set } \mathrm{K}, \\
\mathrm{NA}= & \text { number of data sets with common, given or } \\
& \text { interpolated, measurement in groups } \mathrm{I} \text { and } \mathrm{J}, \\
\mathrm{NA}= & \text { number of data sets with measured or interpolated } \\
& \text { cross sections in group } \mathrm{I} \text { or } \mathrm{J} \text {, whichever is } \\
& \text { greater. }
\end{aligned}
$$


If there are not common data sets in groups $I$ and $J$, the covariance is set to zero. If there are not data inside a group, the standard deviation for that group is set to the average of the corresponding values in neighboring groups.

Step 6: The program also calculates the correlation matrix $[\operatorname{COR}(I, J)]$ according to the formula

$$
\operatorname{coR}(I, J)=\frac{\operatorname{cov}(I, J)}{\sqrt{\operatorname{cov}(I, I) \operatorname{cov}(J, J)}} .
$$

\section{DESCRIPTION OF THE INPUT-OUTPUT SPECIFICATIONS}

\section{A. Input}

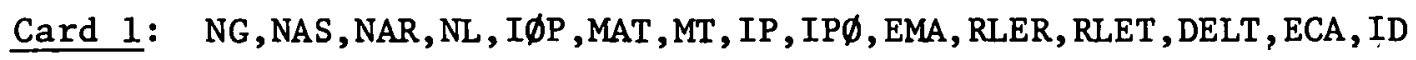
[FORMAT $(9 \mathrm{I} 4,5 \mathrm{E} 7.0, \mathrm{I} 1)]$

NG: number of group;

NAS: number of cross-section data sets included in the error file evaluation;

NAR: number of ratio data sets included in the error file evaluation;

NL: number of energy limits preset to make linear interpolation between measurements in a given data set;

IØP: option

$=0$, the word FISSION is printed;

= 1, the word CAPTURE is printed;

$=3$, the word ALFA is printed;

MAT: material number according to ENDF/B specifications; 
MT: reaction-type number according to ENDF/B specifications;

IP: option

$=0$, part of the input is a cross-section ratio;

$\neq 0$; otherwise;

IP $\emptyset:=1$, the output includes punched cards of the covariance matrix , according to ENDF/B format;

$\neq 1$, otherwise;

EMA: higher energy 1imit corresponding to group 1 (MeV) (group 1 is the highest energy group);

RLER: lethargy corresponding to the lower limit of group 1 (it is assumed that lethargy zero corresponds to $10 \mathrm{MeV}$ );

RLET: , group lethargy ințerval;

DELT: (MeV) increment corresponding to the first energy decade in the decimal group structure;

ECA: (MeV) higher energy limit of the decimal group structure; if all the groups are decimal, EC̣A muṣt be greater than EMA; if all the groups are of constant lethargy ECA must be lower than the lower limit of the last group;

ID: $\neq 0$, the output includes punched cards of the correlation matrix;

$=0$, o.therwise.

Carls 2: $\operatorname{NPE}(\ell) ; \ell=1, \mathrm{NA} ; \mathrm{NA}=\mathrm{NAS}+\mathrm{NAR},[$ FORMAT $(1415)]$ Number of experimental points of set $\ell$.

Cards 3: FORMAT (7E10.0)

Cross sections corresponding to the data set $\ell$. 
Cards 4: FøRMAT (7E10.0) (MeV)

Energies corresponding to the previous data set.

Cards 3 and Cards 4 must be repeated in a sequential order from $\ell=1$ to $\ell=$ NAS. Following these cards, one has to place a set of cards in the same format but now for the ratio measurements (only present if IP $=0)$.

Cards 5: ENDF/B evaluated cross section for group $J(J=1, N G)$ [FØRMAT (6E12.0)]

Cards 6: (Only present if IP $=0$.$) FORMAT (6 \mathrm{E} 12.0)$, ENDF/B evaluated cross sections for group $J(J=1, N G)$ of the reference standard cross section corresponding to the ratio measurement. (Tt is assumed that all ratio measurements in the input are relative to the same cross section.)

Cards 7: FORMAT (7E10.0)

Weight corrcoponding to each data ser $\ell,(\ell=1, N A)$.

Cards 8: FøRMAT (7E10.0). Energy limits for the interpolation procedure (see step 4 in Sect. III). These limits are preset by the user, based on' his judgment on the validity of the interpolation scheme. The liults must be given in MeV and in decreasing $\operatorname{order}[E L(J), J=1, N L]$. 
B. Output

Printed output:

(1) All the measured cross sections are ordered and displayed according to authors and group limits.

(2) The evaluated and the measured cross sections (now reduced to the mean energy of the group interval) are ordered and displayed according to authors and group limits.

(3) The covariance matrix.

(4) The relative standard deviation.

(5) The information contained in each punched card corresponding to the covariance matrix in the ENDF/B format.

(6) The information contained in each punched card corresponding to the correlation matrix. The formats are the following: First NG cards (three values in each card): Lower energy limit (MeV) in group I, and the corresponding relative standard deviation in percent and reference cross sections (1X,E11.4,11X,E11.4,F11.4)

The rest of the cards:

$\operatorname{COR}(\mathrm{I}, \mathrm{J})$, for each $\dot{\mathrm{I}}, \mathrm{J}$ varies from 1 to NG format $7 E 10.4$. These cards are suitable for a three-dimensional plot of the correlation matrix.

(7) The correlation matrix is printed as can be seen in Appendix B :

\section{Punched card:}

With the option (IPO = 1), the covariance matrix is punched out according to the ENDF/B format. 
The correlation matrix output is punched out, under option.

\section{LISTING OF THE PROGRAM AND SAMPLE PROBLEM}

Data for the sample problem: $\left[\operatorname{Cov}(I, J)\right.$ for the ${ }^{240} \mathrm{Pu}$ capture cross section].

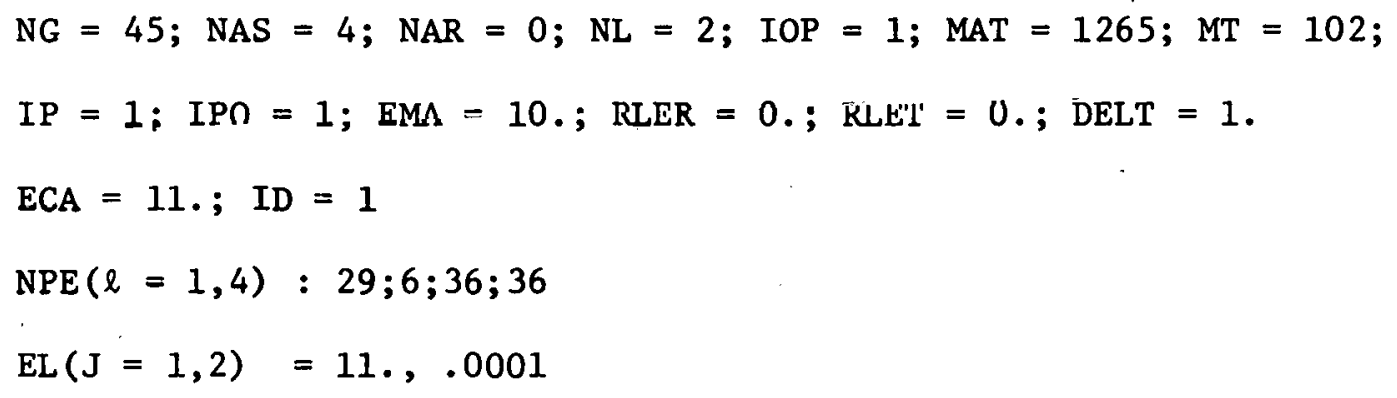




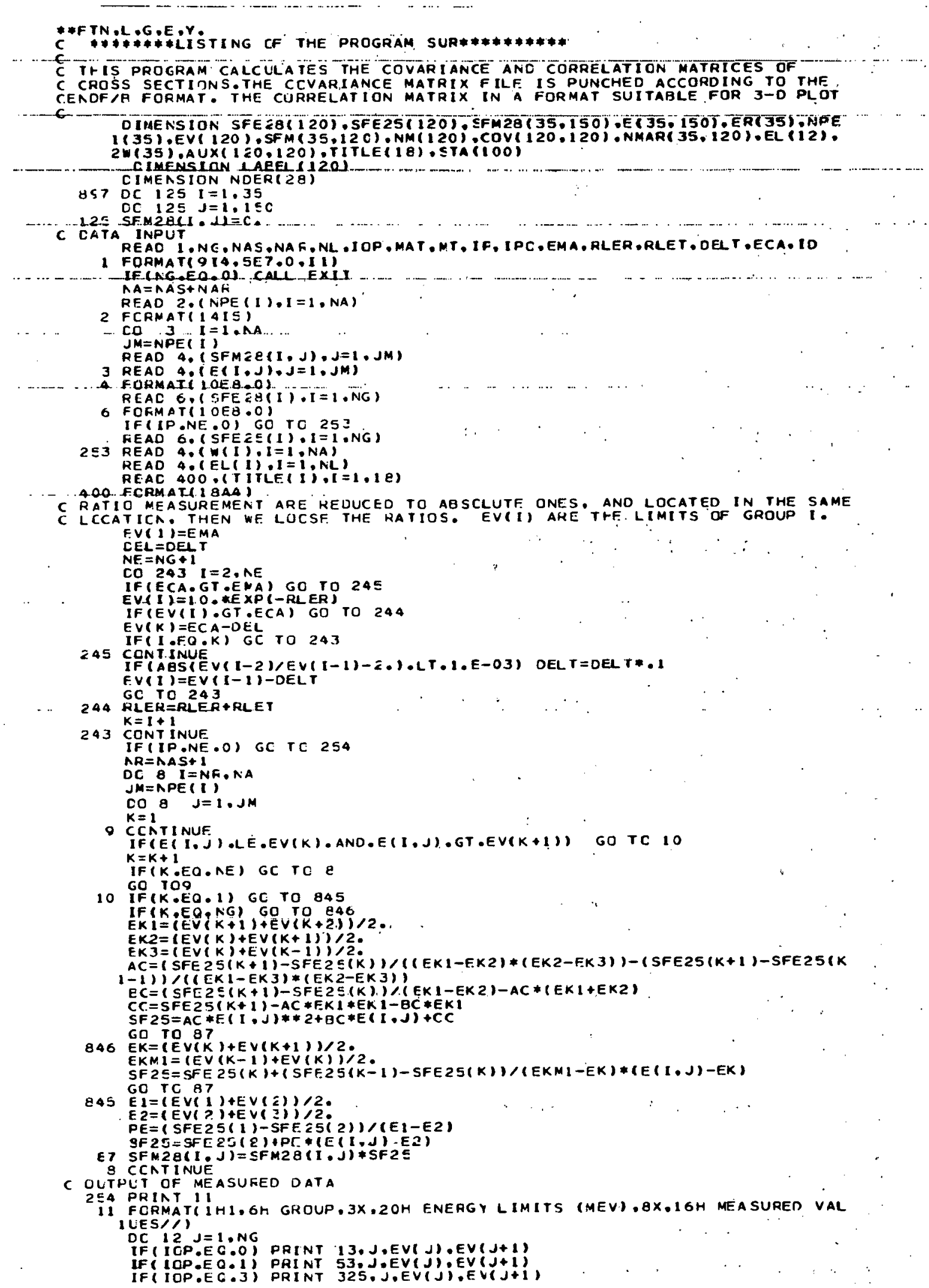


13 FRRMATII6.3X, IPE $11.4 .3 X, 1$ PE 11.4.3X.7H AUTHOR.3X.7H ENERGY.7X.11H S 1 IGF ISSION.7N DEIGHT)

E F FRMAT(IE:3X,IPEII:4.3X.1PEII.4.3X.7H AUTHUR.3X.7H ENERGY.7X.1IH S ATRT. OTH WEIGHT)

1 FORMAT( I6.3X. IPE II:4

LCO $14 \quad I=1$. NA

KN=AFE (I)

DO $14 K=1 . K M$

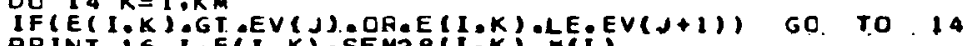

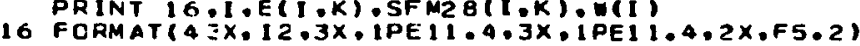

L $=1$

14 CCNTINUE.

IF(L.EO.O) PRINTIIT

17 FOFMAT( $43 \times 1$ SH NC MFASUFEMENT)

12 CONTINUE

C THE MEASUREMRNT CF AUTHOH I INSIDE GFOUP J IS RECUCED TO THE. MEAN. ENERGY OF

THF GROUP AND THEN AVERAGED. THE RESULS ARE LOCATED IN SFM(T;O)

OO 1 e $J=1.12 \mathrm{C}$

$N M(J)=0$

DO $18 \quad 1=1 \cdot 35$

8 SFM(T.aj) $=0$.

CQ $19, I=1, N A$

$K M=A F F$ I

DC $19 \mathrm{~J}=1 . \mathrm{NG}$

KR $=0 \cdot 2: K=1 \cdot K \bar{K}$

IF(E (I.K).GT.EV(J).OR.E(I.K).LE.FVIJ+IS) GO TO 20

IF $(J+E O-i) G G$ TQ 364

IF J J EO N

$E J 2=(E \vee(J)+E \vee(J+1)) / 2$.

$F J z=(E \vee(J)+E \vee(J-1) 1 / 2$.

$A C=(S F E 28(J * 1)-S F E 2 B(J)) /((E J 1-E J 2) *(E J 2-E J 3))-(S F E 28(J+1)-S F E 2 A(J$

$1-1) i((F J)-E J 3) *(E J 2-E J 3))$

$E C=(\operatorname{SFE} 2 \theta(J+1)-S F E 2 E(J)) /(E J I-E J 2)-A C *(E J I+E J 2)$

$C C=S F E 2 B(d+1)-A C \# E J 1 * E J 1-B C * E J 1$

$R K=S F M 2 B(1$ oKi $(A C C E(1 . K)+2+B C+E(I . K)+C C)$

$A C=R K * A C$

$B C=R K * R C$

SF MII.J) $=S F N(I, J)+A C * E J 2 * 2+B C * E J 2+C C$

FR=RR+1. GOTRR+1

355

$E J=(E \vee(J+1)+E \vee(J)) / 2$.

EJMI = (EVI $)+E V(J-1) 12$

$P E=(S F E ? B i J-1)-S F E 2 E(j) i(E j N i-E)$

$\operatorname{SFM}(I, J)=S F M(I, J)+\operatorname{SFM} E(I, K)-P E+(E(I, K)-E J)$

$R R-R R+1$.

GC TC 20

$354 \quad E 1=(E \vee(1)+E \vee(E)) / 2$

$E Z=\{E V(2)+E V(I) ;)<$

$P E=(S F E 2 B(1)-S F E 2 B(2)) /(E 1-E 2)$

SFM(I.I) SFM $(I, I)+S F M 2 B(I \cdot K)-\operatorname{PE} *(E(I, K)-E I)$

FRERR+I

20 CENTINUF

IFIHR $. G T \cdot 0,1 \quad \operatorname{SFM}(I, J)=\operatorname{SFM}(I, J) / R R$

C NUMEER OF REAL MEASURFMENTS NM(J) INTC EACH GROUP

DO $21 J=1, N G$

AMAR $(1, J)=1$

IFISFMi I. J

a 1 CONTINUE

C TIO GROUP ANO ALTHCRS IN WHICH THEGE ARF NO MEASUREMENT ARE MARKED

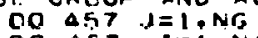

IF (SFM( I.J) EG. O.) NMAR $(I, J)=0$

457 CONTINUE

I MEASURENERT IN GROLP, GY INTERPOLATION

(IF POSSIALE)
CC 110 J=1.NG
IF $J$ JEO. $1.0 F . J . E O . N G)$ CO TO 110

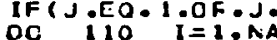

IF(SFMII.J):AE.O.) GO TO 110

$L=1$

111

IF(EV(J).LE -EL(L).AND.EV(J).GT.EL(L+1)) GO TO 12

$\mathbf{L}=\mathbf{L}+\mathbf{1}$

12 KAR =

112 KAR = Ji

IF (SFMII.KAR) .NE.0.) GO TO 114

KAF $=$ KAR - I

IF (KAR.EC.O) GO TO 110

IF(EV(KAF) LE GOL (L) GC TO 113

114 IF(EV(KAR).ET ELL(L)) GO TO 110

KAB $=J+1$

1 IS CGNT INUE

IF(SFM(I.KAE).NE.O.) GO TO 116

$K A E=K A B+i$

IF(KAB.GT.NG) GO TO 110

IFIEV(KAB).CT .EL $(L+1)$ GO TO 115 


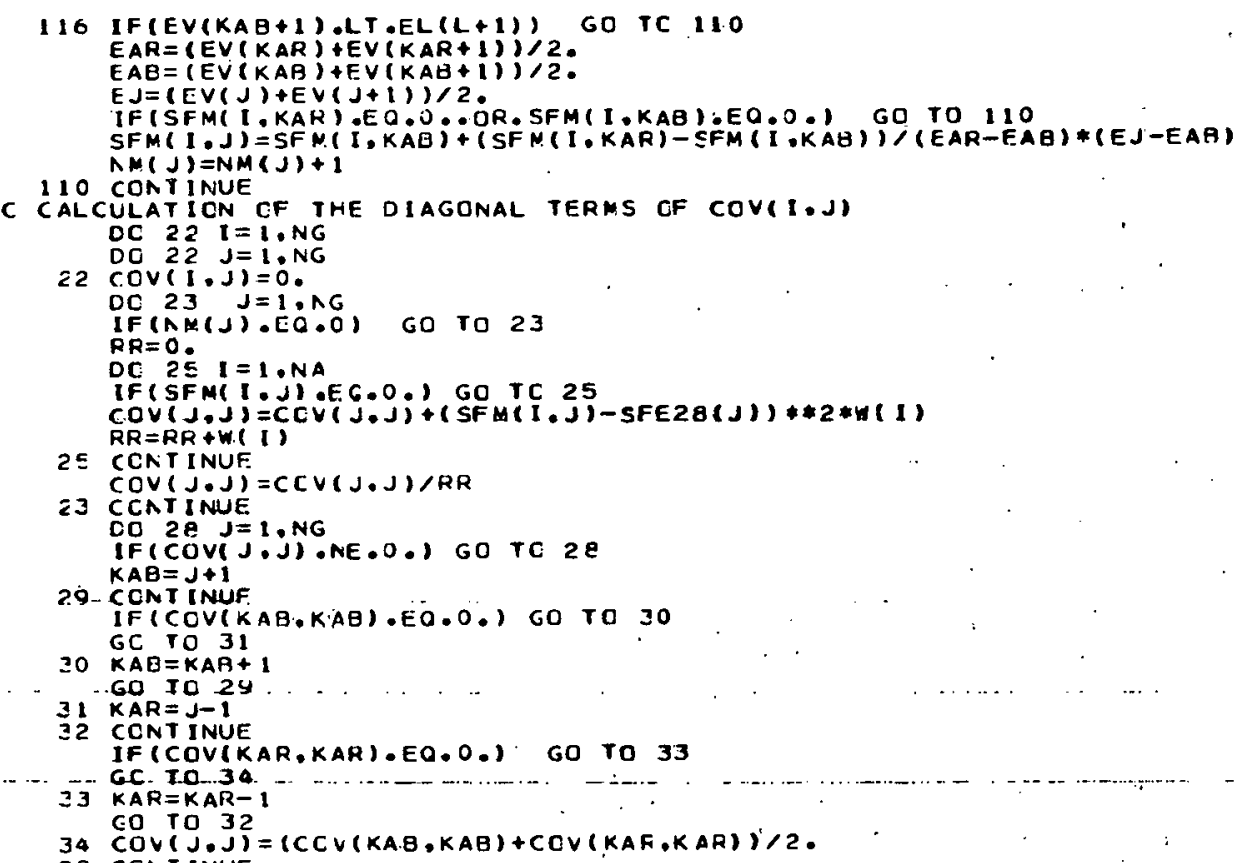

35 FORMATK IHL.6H GROUP, 3X.10H EVALUATEO.3X,6OH MEASURES AND INTERPOLA

1 TED VALUES RECUCED To THE MESA ENERGY/ 1 TEO VALUES RE

IF (ICP.F. C.O P) PRINT 37.J.SFE2B(J) IF (ICP.F.C.0) PRINT 37 J J SFE 28 (IOP.EC:I) IF (IOP.EC:1) PRINT 54.J.SFE2E(J)

32 FORAX

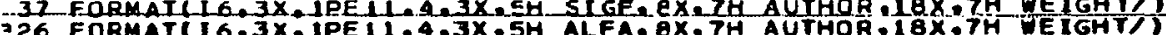

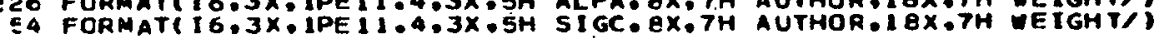
IFIAMPJ) FO. C) PRINT. 38

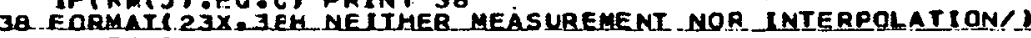
DO $36 \quad I=1 . N A$ IF (SFM(I, J) JE C.0.) GO TO 36 IF(NMAR(I.J).EQ.O O) PRINT 39.SFMII.J),I.W(I)

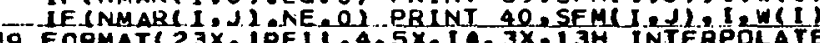

39 FORMATI $23 X$. IPEI $1.4,5 X, 19,3 X, 13 H$ INTERPOLATED, $2 X_{0}, F 5.21$

40 FORMATI $23 X$. IPEII:4.5X,I $4: 3 X, 9 H$ MEASURED.6X,F5.2)

36 CONTINUE

C.CALCULATION OF_CCVCI.UZ MIIH I.NE.J

CO $4 I \quad I=I$, NG

CO 4 I $J=1: N G$

RR $1=0$.

IF (I.EQ.J) GO TO 41

Q $22 K=1 . N A$

(SFM(K.I) EG, O.OOR.SFM(K,J) EO..0.) GO TO 142

COV(

42 IF ISFM(K.I) $N$ NE.0.) RRII=RRI + W(K) IF (SFM(K,J) $\cap$ NE. O.) RAJ $=R R J+M(K)$

42 CONT INUE

IF (RRI,GE ARFJ) RR=RRI

IF (RRI LE RRRJ) RR=RRJ

IF (RR . NE . 0.) COV $(1, J)=C$ CV $(1, J) / R A$

C. OUTPUT OE DATA PRIAT 499

4 SG FORMAT( 1 H1)

DO $357 \quad t=1$, NG

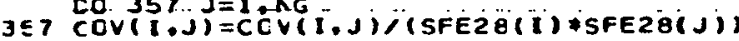

DO $43 I=1, N G$ PRINT 44 . I. NG

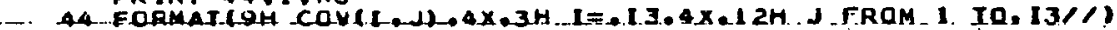
PRINT 45 , (CCVII:J):J=I.NG)

45 FCFMAT(IPIOE12.4)

45 FCFMAT 43 C.CATINUE

C.CAT I NUE

DO $210 \quad I=1 ., \mathrm{AG}^{\circ}$

210 EV(I) $=10000 \mathrm{CO}$ * * V V(I)

E $O=.00001$

EM=2000.00.00.

$N \mathbf{I}=0$

$F I=0$

MIA $=\geq 3$

$R \backslash A=1+$

C THE CRDER IN THE GROUPS ARE INVERTEO 


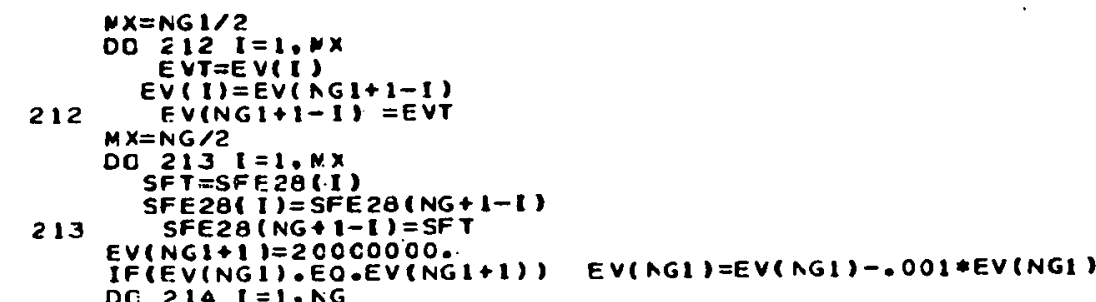

11 GRRM IOW INVERTED/O35H COVII,JI CAROS IN THE ENDF/B FORMAT/

$N 3=N G+3$

$\mathrm{LB}=3$

$A+1+20140+10$

$N 7=N G+7$

OO $216 J=1, N G$

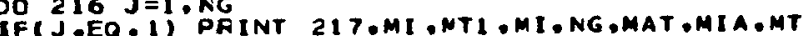

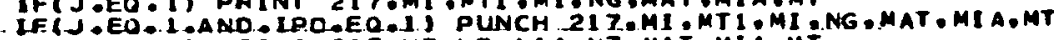

IF (J.GT - I 'PRINT $217 . N 3$, LB,NI4,N7, MAT, MIA.MT

217 FORMAT $(22 X, 4111,14,12,13)$

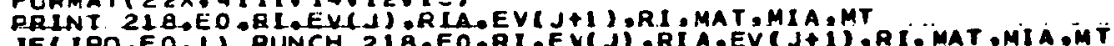

218 FORMATI6 EII $4.14,12.13)$

PRINT $210, E M-R I$ EO ORI EV (1) AUX(1,J) MAT OMIA MT

PRINT

E19 FCRMAT(6FI1.4.14.12.13)

$N G 2=N G+2$

$1=\mathbf{2}$

221. CONISAUE ORT

1 RIA MT O D

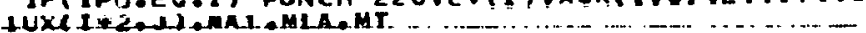

220 FORMAT(OE11.4.14.12.13)

$I=I+3$

IF( (NG2-I).GE.2) GO TO 221

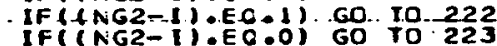

Gn In ? 30

222 PRINT $22 A, E Y(I), A U X(I, J), E V(I+1)$ AUXX $(1+1, J)$ MAT M MIA.MT

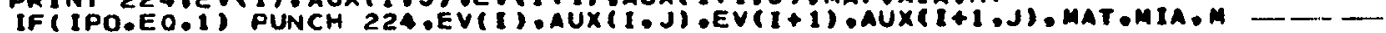

224 FORMATI 4E11.4.22 $\times 1,14,12 \cdot 13)$

223 GR TO 230 , ERT (I), AUXI I, J),MAT,MIA,MT

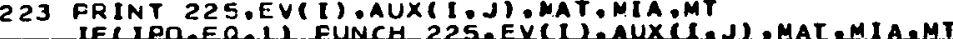

22E FORMATI2E11.4,44X,14.12.13)

230 CONTINUE

216 CCNT INUE

IE ID.ME.OS DUNC

PRINT 3 I 00

310 FORMATIIHI.4IH CORRELATION MATRIX CAROS FOR THE DRAWING/I

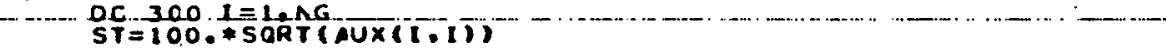

ENE $=.000001$ *EV(I)

PRINT 301 IENE ST

IEC

3 CO FOFMATC 1 CONT INUE

EAE =EV(NE) I $10 * * 6$

ENE

DO 311 I $=1 . N G$

$321 \operatorname{COV}(1, \lambda)=A U \times(1, \lambda) / \operatorname{SQRT}(\operatorname{AUX}(1,1) * \operatorname{AUX}(J, J))$

DO 302 I $=1$. AG

PRINT $305,($ (CCV (I,J) J J $=1$ NG $)(J), J=1, N G)$

3 C FORMAT 8 F9.5)

30 EORMATIIX.TEIO.4

305 CORMATR

DO $391 \mathrm{I}=1, \mathrm{NG}$

391 STACI $=100$ * SCRT (AU X(1,I)

$4 C 2$ FORMAT (IHI.1BAA)

3 CO $399 N=2,2 E$

35. OC $400-1=1 . M G$ 


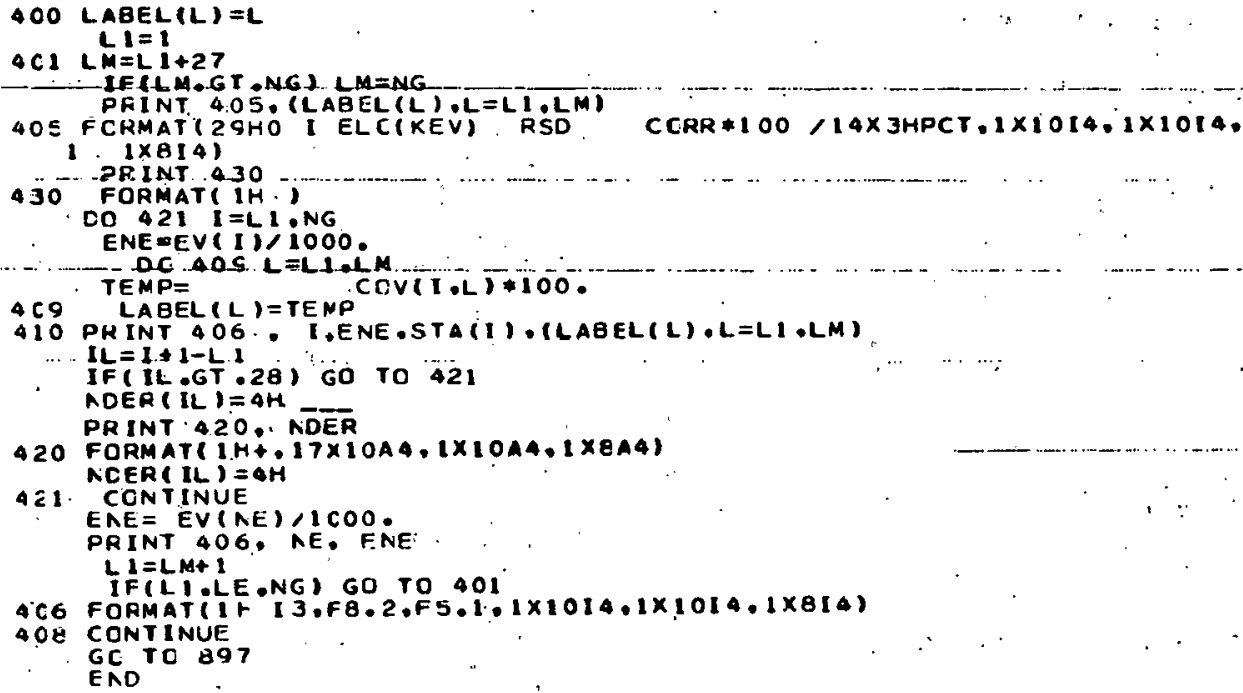


GROUP ENERGY LIMITS (MEV)

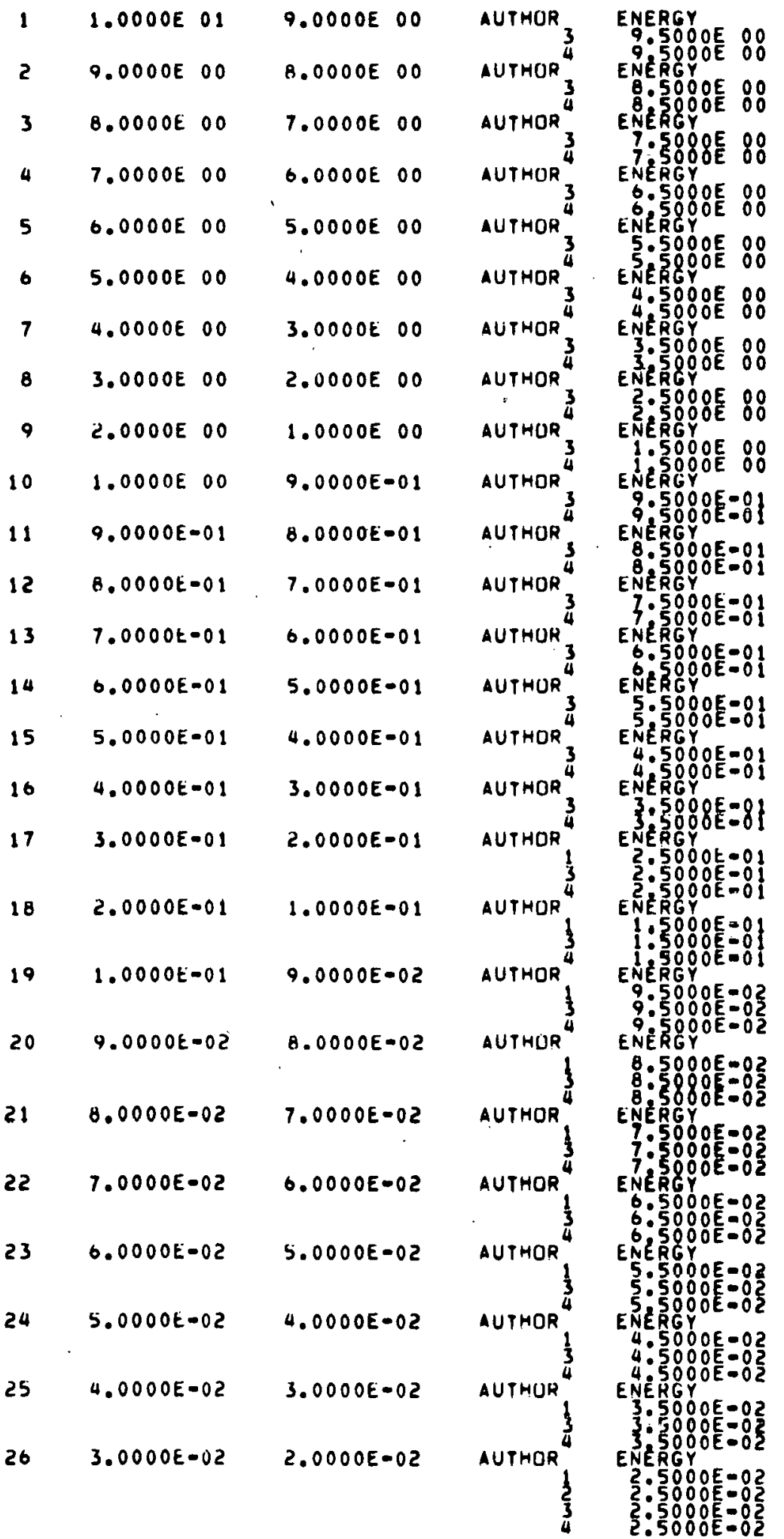

SIGCAPTURE MEIGMT $4.7000 E-03$ T.00 SIGCAPTURE WEIGATO $5.70000-03 \quad 1.00$ 5 I 3000 ETOO 3 WEIGATO $7.0000 E-03$
600000
3 8.3000 - 33 WEIGHT 1.00 STECAPTURE WEIGAT SIGEAPTURE WEIGAT SIEEAPFURE WEIGATO $1.6000 E-02 \quad 1.00$ SIGCAPTYRE WEI GHT $2.4000 \mathrm{E}=02 \quad 1.00$ SIECR\&PURE WEIGATO 4.4000500 ? 1.00 SIGCAPTURE WEIGAT $7.3000 E-02 \quad 1.00$ SIECAPYURE WE IGATO

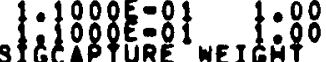
SI GOPOORE, WEIGHTO SIECAPYURE WEIGAT SIGCAP OUR WEI IA 1 15000E-01 1.00 SIGCAPJYRE WEIGHT SECOROEYR WEIGAOO 1.5000 501 T.00 SIGCAPTURE WEIGATO $1.6000 E=01 \quad 1.00$ SIECAPPURE WEIGAOT 3.3888:-81 $1: 00$ SIECAPTURE WEIGHT $1: 80000^{-01} 1: 00$ FEOOOEOO WEI S 520000 SISCAPYURE WEIGA $3.4600=02$ WEI.00 2:70000=01 $\quad 1: 80$ SIGCAP\&URE WEIGH $3.8200 E-01 \quad 9.00$

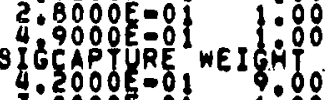
$3.0000=0$ SFECAPYURE WEIGAO $4.6800 E=01$. 9.00 5.3000E-01 1.00 SIECAPTURE WEIGHT $5.8300=01$ Q 9.00 6 SOPOE-O WEIGAO ISCAP JURE, WEIGHTO $4.2000 E-01 \quad 1.00$ SIGCAPTURE WEIGHY $6.9100=01$ 9. 988 80000 - 1 1 SIECAPTURE WEIGHT $8.9000 E=1 \quad 900$ $5: 5000=01 \quad 1: 00$ 


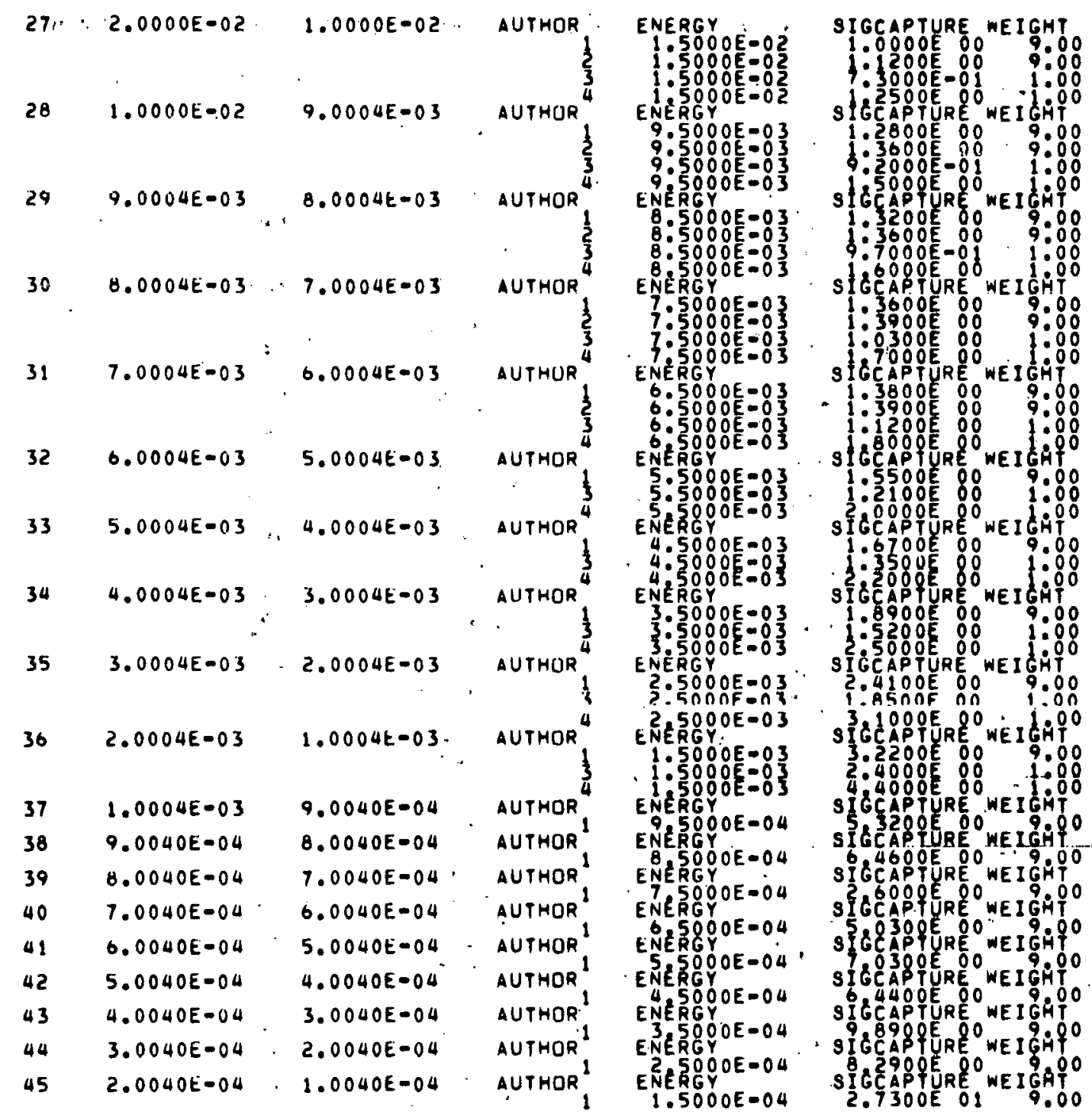


gruUP EVALUATED MEASURES AND INTERPOLATEO VALUES REDUCEO TO THE MESN ENERGY

\begin{tabular}{|c|c|c|c|c|c|}
\hline 1 & $5.1000 E=03$ & SIGC & AUTHOR & & WEIGHT \\
\hline 2 & $5.8000 E-03$ & $\begin{array}{l}4.7000 E=03 \\
47000 E=03 \\
91 G C\end{array}$ & AUTHOR & $\begin{array}{l}\text { MEASURED } \\
\text { MEASURE.D }\end{array}$ & $\begin{array}{l}1.00 \\
1: 80 H T \\
W E I G H T\end{array}$ \\
\hline 3 & $0.9000 E-03$ & $\begin{array}{l}5.7000 t=03 \\
53000 E=03 \\
\text { S16C }\end{array}$ & AUTHOR & $\begin{array}{l}\text { MEASURED } \\
\text { MEASURED }\end{array}$ & HE \\
\hline 4 & $8.5000 E-03$ & $\begin{array}{l}7.0000 E=03 \\
800000 E=03 \\
\text { SIGC }\end{array}$ & AUTHUR & $\begin{array}{l}\text { MEASURED } \\
\text { MEASURED }\end{array}$ & $\begin{array}{l}1.00 \\
1.00 \\
\text { WEIGHT }\end{array}$ \\
\hline 5 & $1.1000 E-02$ & $\begin{array}{l}8.3000 E=03 \\
71600 E=03 \\
\text { SI } 10 \mathrm{C}\end{array}$ & AUTHOR & $\begin{array}{l}\text { MEASUREO } \\
\text { MEASURED }\end{array}$ & $\begin{array}{l}1.00 \\
\text { LEIOHT }\end{array}$ \\
\hline 6 & $1.5000 E-02$ & 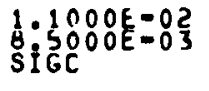 & AUTHOR & $\begin{array}{l}\text { MEASUREO } \\
\text { MEASUREED }\end{array}$ & $\begin{array}{l}1.00 \\
\text { WEIGHT }\end{array}$ \\
\hline 7 & $2.4000 E-02$ & $\begin{array}{l}1.6000 E=02 \\
\$ 16800 E=02\end{array}$ & AUTHOR & $\begin{array}{l}\text { MEASURED } \\
\text { MEASURED }\end{array}$ & $\begin{array}{l}1.00 \\
1 E 9 \ell_{H T}\end{array}$ \\
\hline 6 & $4.0000 E-02$ & $\begin{array}{l}2.4000 E=02 \\
\$ 19000 E=02 \\
S 1 G C\end{array}$ & AUTHOR & $\begin{array}{l}\text { MEASURED } \\
\text { MEASUREU }\end{array}$ & WEI \\
\hline 9 & $7.5000 E-02$ & $\begin{array}{l}4.4000 E=02 \\
33000 E=02 \\
\text { SIGC }\end{array}$ & AUTHOR & $\begin{array}{l}\text { MEASURED } \\
\text { MEASURED }\end{array}$ & $\begin{array}{l}1.00 \\
\text { WEIGHT }\end{array}$ \\
\hline 10 & $1.1200 E-01$ & $\begin{array}{l}7.3000 E=02 \\
684000 E=02 \\
\text { SI GL }\end{array}$ & AUTHOR & $\begin{array}{l}\text { MEASURED } \\
\text { MEASURED }\end{array}$ & $\begin{array}{l}1.00 \\
1 E Q 0 \\
\text { WETT }\end{array}$ \\
\hline 11 & $1.2300 E-01$ & $\begin{array}{l}1: 1000 E=01 \\
1000 E=01 \\
\text { SIGC }\end{array}$ & AUTHOR & $\begin{array}{l}\text { MEASURED } \\
\text { MEASURED }\end{array}$ & $\begin{array}{l}1.00 \\
1.00 \\
\text { WEIGHT }\end{array}$ \\
\hline 12 & $1.3300 E=01$ & $\begin{array}{l}1.2000 E=01 \\
\$ 12000 E=01 \\
\text { SIGC }\end{array}$ & $\begin{array}{r}3 \\
4 \\
\text { AUTHUR }\end{array}$ & $\begin{array}{l}\text { MEASURED } \\
\text { MEASURED }\end{array}$ & $\begin{array}{l}1.00 \\
\text { IEOOGHT }\end{array}$ \\
\hline 13 & $1.4400 E-01$ & $\begin{array}{l}13000 E=01 \\
\$ 16000=01 \\
\$ 1 G 8\end{array}$ & AUTHOK & $\begin{array}{l}\text { MEASURED } \\
\text { MEASURED }\end{array}$ & $\begin{array}{l}1.88 \\
\text { WE }\end{array}$ \\
\hline 14 & $1.5300 E-01$ & $\begin{array}{l}1.4000 E=01 \\
19000 E=01 \\
\text { SIGC }\end{array}$ & AUTHOR & $\begin{array}{l}\text { MEASURED } \\
\text { MEASURED }\end{array}$ & $\begin{array}{l}1.00 \\
1.00 \\
W E I G H T\end{array}$ \\
\hline 15 & $1.6100 E-01$ & $\begin{array}{l}1: 5000 E=01 \\
21000 E=01 \\
\text { SIGC }\end{array}$ & AUTHUค & $\begin{array}{l}\text { MEASURED } \\
\text { MEASURED }\end{array}$ & $\begin{array}{l}\text { L.0O } \\
\text { WEIGOHT }\end{array}$ \\
\hline 16 & $1.6500 E-01$ & $\begin{array}{l}1.6000 E=01 \\
2: 3000 E=01 \\
\text { SIGC }\end{array}$ & AUTHOR & $\begin{array}{l}\text { MEASURED } \\
\text { MEASURED }\end{array}$ & $\begin{array}{l}1.00 \\
1: O_{0} \\
\text { WESTH }\end{array}$ \\
\hline 17 & $1.8200 E-01$ & $\begin{array}{l}1.6000 E \\
25000 E \\
\text { S1GC }\end{array}$ & AUTHOR & $\begin{array}{l}\text { MEASURED } \\
\text { MEASURED }\end{array}$ & $\begin{array}{l}\text { WEIGH } \\
1.00 \\
1.00 \\
\text { WEIGH }\end{array}$ \\
\hline 18 & $2.2200 E-01$ & $\begin{array}{l}2.0200 E=01 \\
1.8000 E=01 \\
288000 E=01 \\
S 1 G C\end{array}$ & AUTHOR & $\begin{array}{l}\text { MEASURED } \\
\text { MEASUREED } \\
\text { MEASURED }\end{array}$ & $\begin{array}{l}9.00 \\
1.00 \\
1.00 \\
\text { WEIGHT }\end{array}$ \\
\hline 19 & $2.7200 E-01$ & $\begin{array}{l}2.9400 E=01 \\
292000 E=01 \\
35000 E=01 \\
\text { SIGC }\end{array}$ & AUTHOR & $\begin{array}{l}\text { MEASURED } \\
\text { MEASURED } \\
\text { MEASURED }\end{array}$ & $\begin{array}{l}9.00 \\
1: 00 \\
\text { WEIg }\end{array}$ \\
\hline 20 & $2.8800 E-01$ & $\begin{array}{l}3.4600 E=0 \\
2.7000 E=0 \\
4.6800 E=0 \\
31 G C\end{array}$ & AUTHOR & $\begin{array}{l}\text { MEASURED } \\
\text { MEASURED } \\
\text { MEASURED }\end{array}$ & $\begin{array}{l}9.00 \\
1: 08 \\
1: 08 \\
\text { WEIGHT }\end{array}$ \\
\hline 1 & $3.1000 E=01$ & $\begin{array}{l}3.8200 E-01 \\
2.8000 E=01 \\
49000 E=01 \\
\text { SIGC }\end{array}$ & AUTHDR & $\begin{array}{l}\text { MEASURED } \\
\text { MEASURED } \\
\text { MEASURED }\end{array}$ & $\begin{array}{l}9.00 \\
1: 00 \\
1: 00 \\
\text { WEIGHT }\end{array}$ \\
\hline 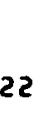 & $3.4100 E-01$ & $\begin{array}{l}4.2000 E= \\
3.8000 E= \\
512000= \\
S 1 G C\end{array}$ & AUTHOR & $\begin{array}{l}\text { MEASURED } \\
\text { MEASURE } \\
\text { MEASURED }\end{array}$ & $\begin{array}{l}9.00 \\
1.00 \\
1: 00 \\
W E I G H T\end{array}$ \\
\hline & & $\begin{array}{l}4.680 \\
3.300 \\
5.700\end{array}$ & $\sum_{4}^{1}$ & $\begin{array}{l}\text { MEASURED } \\
\text { MEASURED } \\
\text { MEASURED }\end{array}$ & $\begin{array}{l}9.80 \\
1.00 \\
1.00\end{array}$ \\
\hline
\end{tabular}




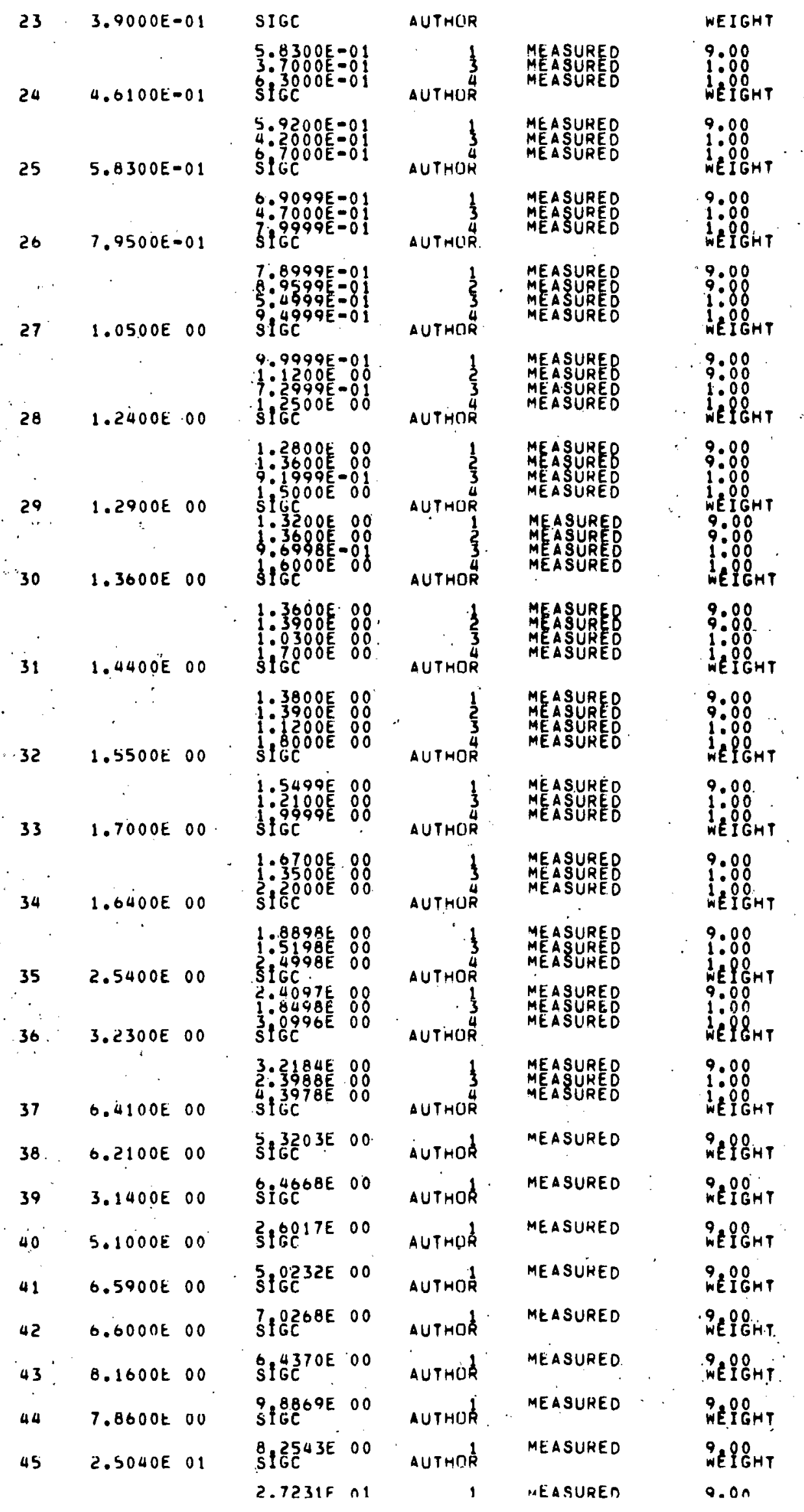




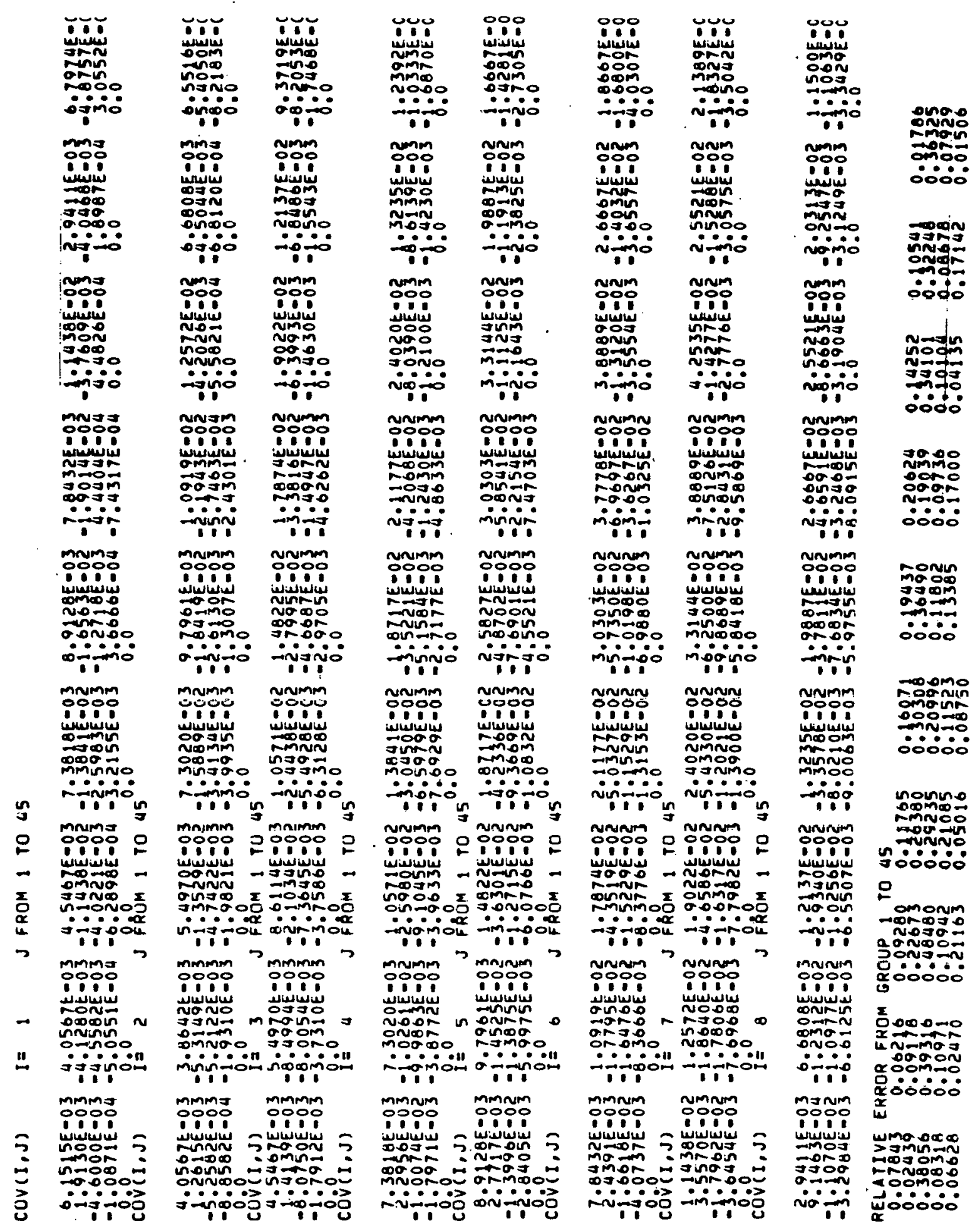


CAROS OUTPUT, WARNING THE ORDER IN THE GROUPS ARE NOW INVERTED COV $(I, J)$ CARDS IN THE ENOF/B FORMA
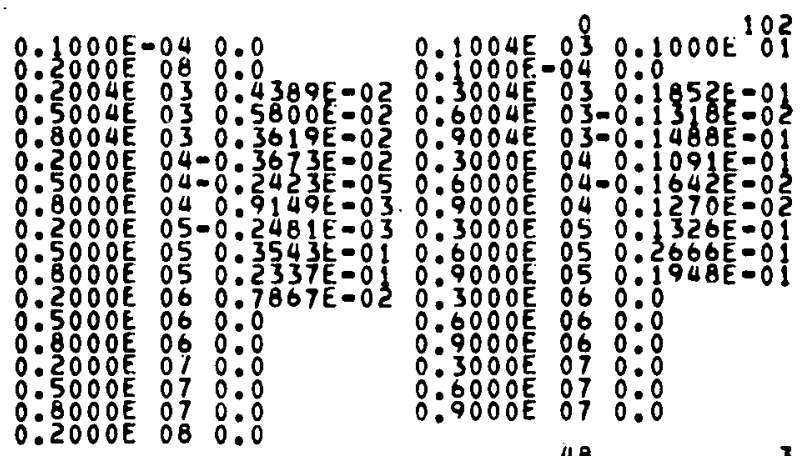

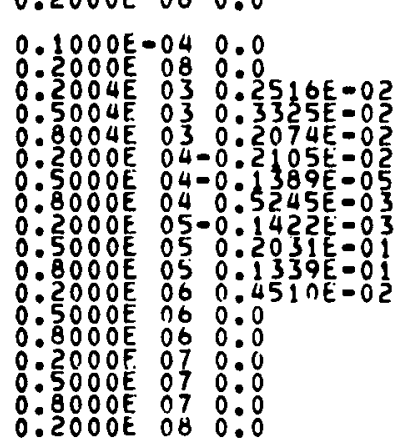

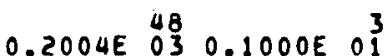
$0: 20000=04 \quad 0: 100$ 0

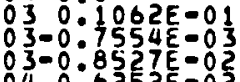
0.9004 E $0.6000 \mathrm{E}$

$040.8527=02$ 作 $0: 3000$ : $050: 7602 \ldots=02$ $0: 9000$ O5 $0: 11117=01$ $0: 3000$ E6 $0: 0$

$0: 60000$ E 0600

$090000^{0} 0: 0$

$8: 30000 \% 078: 0$

$0.1000 E-04 \quad 0: 0$ $0: 200$ UE $03 \quad 0: 1002 E=01$

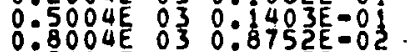

$0.3004 E_{03}^{48} 0.1000 E^{3}$ O1 $0.2004 E$
$0: 1804 E$
$0: 4004 E$
$0: 7004 E$ $0 \begin{array}{r}45126533102 \\ 033009656 E-02126533102\end{array}$ $0: 40005$ $0: 7000 \mathrm{E}$

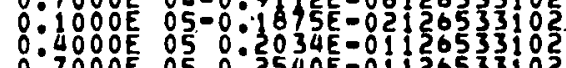
. $050.2540=01126533102$ 0.1000 106 $00.2322 E-01126533102$ 0.000 OP000E 06 :00 80 $0: 40005878: 0$ $0: 1000 E$. $070: 0$

作 .

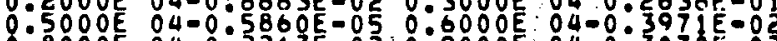
$0.2000^{4}-0.2213 E=0250.9000 E$ O4 $0.3070 E=02$ $0: 5000 E$ 05 0.8569E-01 0:6000E 05 0.6449E-01 $0: 8000 E$ O5 0:5652E-01 0:9000E 05 0:4711E-01 $8: 2000 E 060: 1903 E=01$. 0.8000 Ko 0.0 $0 \quad 0.9000=0000$ $0: 5000$ E $070: 0$ 0.2000 E 08 0:0 0.3000 E 8600 0

$0.3004 E^{10.4}$

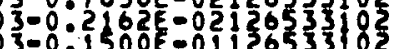
04=-02567E-0 126533105 $4=0.060 .0313653102$ U. $0.4004 E$ $0.7004 E$ $8: 1000$ E $0: 7000$ . $0: 7000$ E

$0: 1000$ E $0: 7000$ E $060: 0$ $0: 1000$ E 07.00 $0: 4000507 \quad 0: 0$ $\begin{array}{lll}8: 7000 E & 07 & 0 \\ 0.1000 & 08 & 0\end{array}$

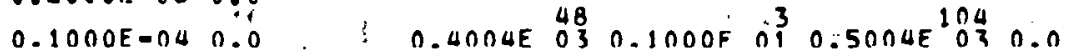

$0.4004 E^{104} 03$

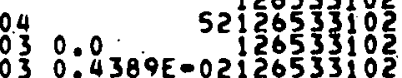

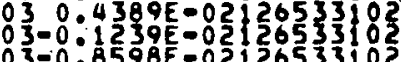

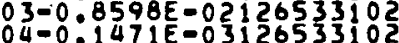
$=0.7243 E=03126533102$ 5 =0:5223E=0612653310 $50.1166=01126533102$ $0.3000 \mathrm{E}-040.100$ $0: 1004 E$

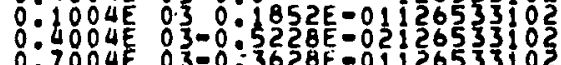
$0.70040^{0}=03628 k=012553103$ .01000 E $04=0: 6200 E=03126533102$

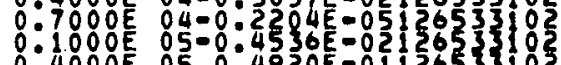
$0: 4000 E$ 05 $0: 4920 E-011265\} 310$ है $0.7000 E 05006144 E=01126533102$

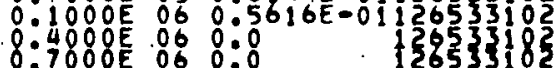
$0: 1000 E 878: 0$ :7008E:07 0.8 $0.1000 E \quad 08 \quad 0.0$ 
CORRELATIUN MATRIX CAROS FOR THE DRAWING

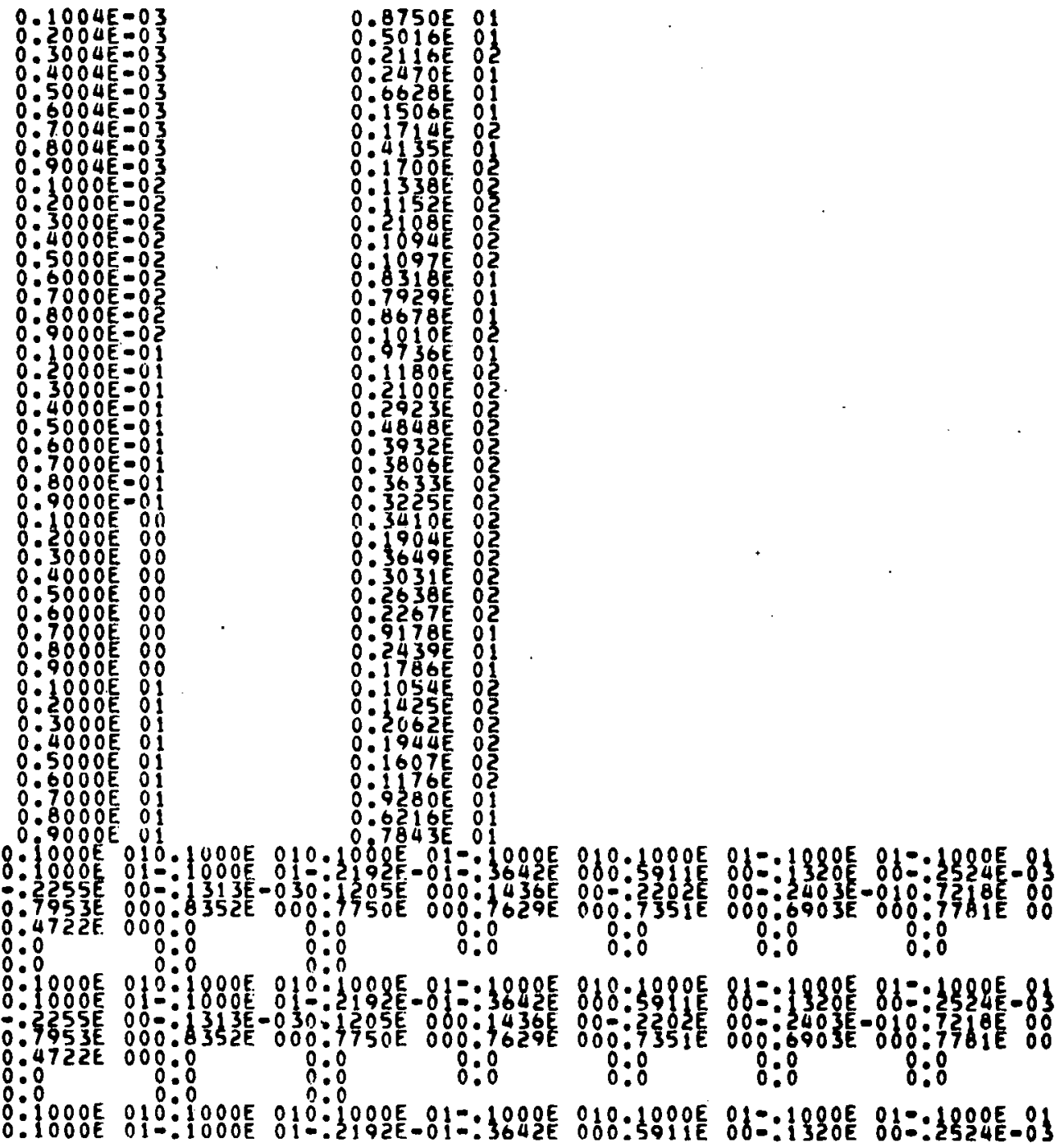




\section{THE THREE-DIMENSIONAL CORRELATION MATRIX PLOT}

As noted, part of the card output of the program can be used to generate a three-dimensional plot of the correlation matrix. This can be seen in Fig. 1, where this plot is shown for the ${ }^{238} \mathrm{U}$ fission cross section.

Several conclusions can be deduced from this plot:

(1) A lot of structure can be seen. This corresponds to the fact that the cross section was analyzed with very much detail.

(2) The standard deviation shows large uncertainties in the low-energy region. This reflects the lack of data in this region.

(3) Positive correlation corresponds to differences in magnitude but not in shape; negative correlation corresponds to differences in shape. Then, differences in shape in the low-energy range can be detected. This reflects the fact that in this zone the cross section rises so fast that differences in cross-section values correspond to a large difference in shape.

(4) The recent measurement of Behrens et al. correlates all the analyzed energy intervals. 9 

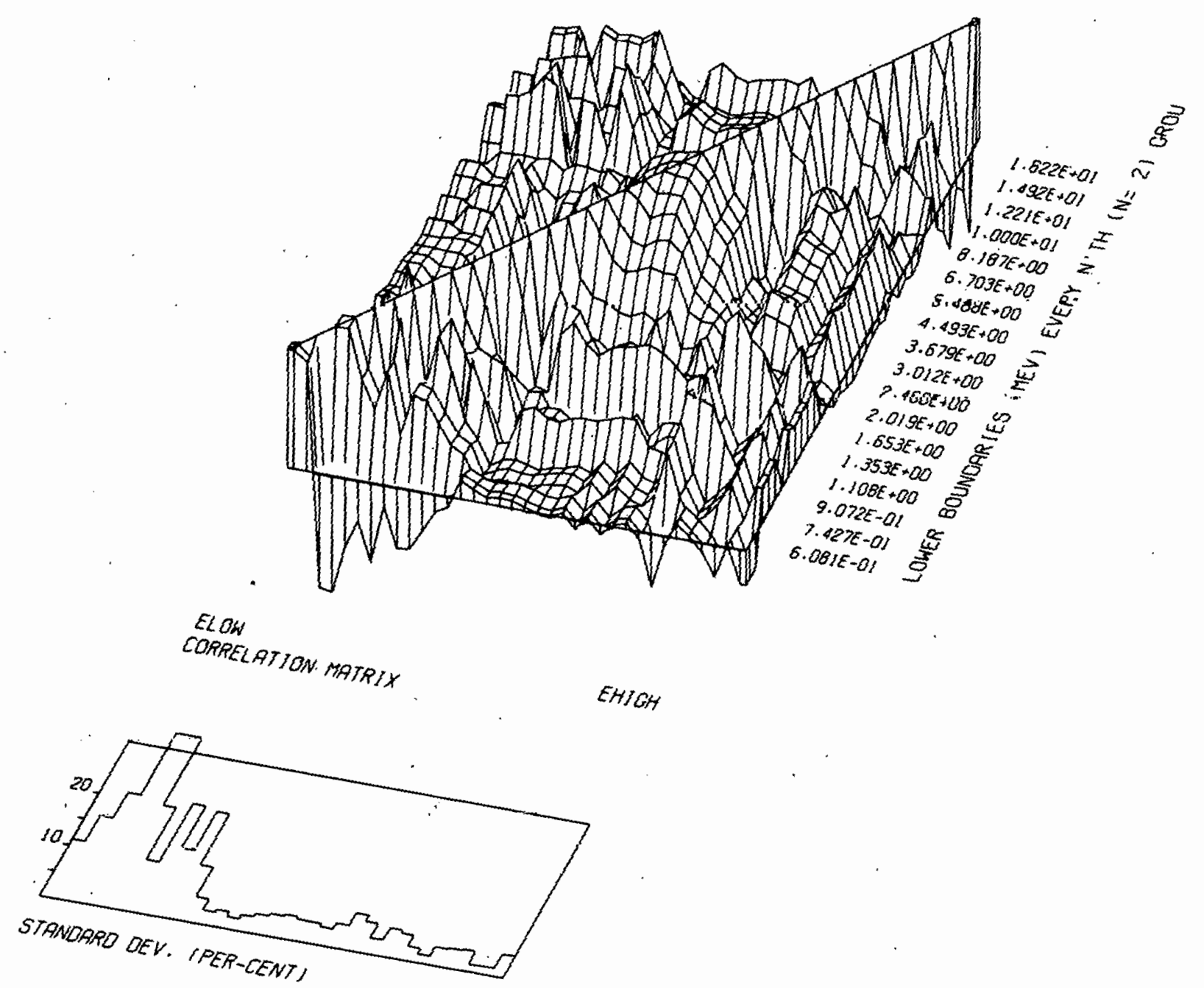

MAT'L 126236 GROUPS RERCT 18 TO REACT 18 


\section{REFERENCES}

1. Greebler, P., et al., "Implications of Nuclear Data Uncertainties to Reactor Design," Nuclear Data for Reactor, Proc. Conf., Heisinki, 1970, IAEA, vol. I, 17.

2. Bäré, Y., et al., "Roles Respectifs des Evaluations et des Experiences Integrales pour la Physique des.Reacteurs Rapides," Applications of Nuclear Data in Science and Technology, Symp., Paris, 1973.

3. Usachev, L. N., et al., "Nuclear Data Sources and Their Implications on the Design of Fast Reactors," Applications of Nuclear Data in Science and Technology, Symp., Paris, 1973.

4. Hummel, H., et al., FRA-TM-54, ANL (1973).

5. Votinen, V. 0., et a1., "The Light-Water Industry. Nuclear Data Needs," Conf. on Nuclear Cross Sections and Technology, Washington, D.C., March 1975.

6. Minutes of the spring and fall 1973 meeting of the cross-section evaluation group and F. G. Perey. "Format and procedures for ENDF/B data covariance files." 
7. Perey, F. G., et al., "Estimated Data Covariance Files of Evaluated Cross Section - Examples for U-235 and U-238," paper presented at ANS meeting, Atlanta, September 1974.

8. Perey, F. G., "Estimated Uncertainties in Nuclear Data. An Approach," Conf. on Nuclear Cross Sections and Technology, Washington, D.C., March 1975.

9. Behrens, J. W., et al., Conf. of Nuclear Cross Sections and Technology, paper GB-14, Washington, D.C., March 1975. 
APPENDIX A. LIST OF REFERENCES USED IN THE CALCULATION.

OF THE COVARIANCE MATRICES

$238^{2}$ Fission: In this case the covariance $[\operatorname{COV}(I, J)]$ was calculated for 36 groups from $20 \mathrm{MeV}$ to $550.24 \mathrm{keV}$ in a 0.1 lethergy unit interval. The following set of references was used:

1. Nyer, W., Report LA-719 (1948).

One measurement at $14 \mathrm{MeV}$. Original data.

2. Uttley, C. A., et al., Report AERE NP/R 1996 (1956).

Two measurements at 14.1 and $15 \mathrm{MeV}$. Original data.

3. Smith, R. K., et a1., Bulz. Am. Phys. Soc. 2: 196 (1957).

Thirty seven measurements at 1-20 MeV. Results from Sowerby, M. G., et al., Report AERE-R7273 (1973).

4. Billand, P., et al., Geneva Conf. 16: 106 (1958).

One measurement at $13.6 \mathrm{MeV}$. Original data.

5. Flerov, N. N., et a1., J. Nucl. Energy 11: 173 (1959).

One measurement at $14.6 \mathrm{MeV}$. Original data.

6. Mangialajo, M., et al., Nucl. Phys. 43: 124 (1963).

Eight measurements at $13.74-14.75 \mathrm{MeV}$. Original data.

7. Barral, R. C., et al., Report AFWL-TR-68-134 (1969).

One measurement at $14.6 \mathrm{MeV}$. Original data.

8. Henkel, R. L., et a1., Report LA-1495 (1952) and Report LA-2122 (1957)

Thirty eight measurements at $1.3-6.92 \mathrm{MeV}$. Data renormalized to $0.5176 \mathrm{~b}$ at $4.50 \mathrm{MeV}$. 
9. Sailor, V. L., Report WASH-745, p. 31 (1958).

Four measurements at $8.8-13.86 \mathrm{MeV}$. Renormalized to $1.109 \mathrm{~b}$ at $13.86 \mathrm{MeV}$.

10. Adams, B., et a1., J. Nucl. Energy 14: 85 (1961).

Fourteen measurements at $12.7-19.4 \mathrm{MeV}$. Renormalized to $1.125 \mathrm{~b}$ at $14 \mathrm{MeV}$.

11. Pankratov, V. M., et al., J. Nucl. Energy 16: 494 (1962). Fifteen measurements at 10.6-20.4 MeV. Renormalized to $1.125 \mathrm{~b}$ at $14 \mathrm{MeV}$.

12. Pankratov, V. M., At. Energ. 14: 167 (1963). Thirty six measurements at. 5.1-22.5 MeV. Renormalized to $1.125 \mathrm{~b}$ at $14 \mathrm{MeV}$.

13. Emma, V., et al., Nucz. Phys. 63: 641 (1965).

The following are ratio measurements relative to the ${ }^{235} \mathrm{U}$ fission cross section. They were reduced to ${ }^{238} \mathrm{U}$ fission data utilizing the $235 U$ tission cross-section ENDF/B-IV evaluation.

14. Nyer, W., Report LA-938 (1950).

One measurement at $14 \mathrm{MeV}$.

15. Jarvis, G. A., et a1., Report LA-1571 (1953).

One measurement at $2.5 \mathrm{MeV}$.

16. Lamphere, R. W., Phys. Rev. 104: 1654 (1956).

Eighty eight measurements at 0.573-2.995 MeV, measurement multiplied by 0.95 . 


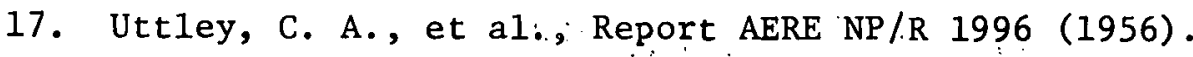

One measurement at $14.1 \mathrm{MeV}$.

18. Kalinin, S. P., et al., Geneva Conf. 16: 136 (1958). Twenty measurements at 2.95-8.30 MeV.

19. Berezin, A. A., et a1., J. Nucl. Energy 11: 175 (1960). One measurement at $14.6 \mathrm{MeV}$.

20. Smirenkin, G. N., et al., At. Energ. 13: 974 (1962). One measurement at $2.5 \mathrm{MeV}$.

21. Stein, W. E., et a1., p. 627 in Proc. Conf. Neutron Cross Sections and Technology, vol. I, Washington, D.C., 1968.

Fourteen measurements at $1.5-5 \mathrm{MeV}$.

22. White, P. H., et al., J. Nucl. Energy 21: 671 (1967).

Three measurements at 2.25-14.1 MeV.

23. Grund1, J. A., Nucl. Sci. Eng. 30: 39 (1967).

Sixteen measurements at $1.68-8.07 \mathrm{MeV}$.

24. Poenitz, W. P., et a1., J. Nucz. Energy 26: 483 (1972).

Three measurements at $2-3 \mathrm{MeV}$.

25. Meadows, J. W., Nucl. Sci. Eng. 49: 310 (1972).

Twenty one measurements al 0.898-5.33 MeV.

26. Behrens, J. w., et al., Conf. of Nuclear Cross Sections and Technology, paper GB-14, Washington, D.C., March 1975. White source, 30.23-0.1077 MeV.

$2{ }^{38} \mathrm{U}$ Capture. In this case the covariance $[\operatorname{COV}(I, J)]$ was calculated for 91 groups for energies ranging from $10 \mathrm{MeV}$ to $100 \mathrm{eV}$. From $10 \mathrm{MeV}$ down to $4.095 \mathrm{keV}, 0.1$ lethargy unit intervals were used. The energy range 
between $4.095 \mathrm{keV}$ and $100 \mathrm{eV}$ was divided in decimal intervals. The following set of references was used:

1. Rose, B., Report AERE NP/R 1743 (1955). See also J. Nucl. Energy 8: 197 (1959).

Eleven measurements at $29-840 \mathrm{keV}$. Original data.

2. Broda, E., et ai., Report BR-754. Reported by Rose in ref. 1. Seven measurements at $400 \mathrm{keV}-4.05 \mathrm{MeV}$. Data revised by Davey in ref. 19.

3. English and Gueron, National Research Council of Canada, Montreal Laboratory Report MC-69. Reported by Rose in ref. 1. Two measurements at $220-850 \mathrm{keV}$. Original data.

4. Macklin, R. L., et a1., Phys. Rev. 107: 504 (1957). One measurement at $25 \mathrm{keV}$. Original data.

5. Lyon, W. S., et a1., Phys. Rev. 114: 1619 (1959). One measurement at $195 \mathrm{keV}$. Original data.

6. Bilpuch, E. G., Ann. Phys, 10:455 (1960). Thirteen measurements at $2.55-217 \mathrm{keV}$. Data revised by Davey in ref. 19.

7. Given, B. G., et al., Phys. Rev. 120: 556 (1960). Seven measurements at $0.175-1 \mathrm{MeV}$. Data revised by Davey in ref. 19 .

8. Gibbons, J. H., et al., Phys, Rev. 122: 182 (1961). Two measurements at $30-65 \mathrm{keV}$. Original data. 
9. Bergginst, I., Ark. Fys. 23: 425 (1963).

Eight measurements at $18-300 \mathrm{keV}$. Data revised by Davey in ref. 19.

10. de Saussure, G., et al., Report ORNL-3360 (1963).

Two measurements at $30-64 \mathrm{keV}$. Data revised by Davey in ref. 19.

11. Macklin, R. L., et a1., Report WASH-1046, p. 88 (1963).

Sixteen measurements at $8.8-54.7 \mathrm{keV}$. Data according to a recent evaluation of $\sigma(\mathrm{Ta})$ in ref. 19.

12. Tolstikov, V. A., et al., J. Nucl. Energy 18: 599 (1964). Eleven measurements at $15-180 \mathrm{keV}$. Corrected by non-1/v $10_{\mathrm{B}}$ behavior and normalized to the Belanova (ref. 13) value. Data from Sowerby. (ref. 20).

13. Belanova, T. A., et al., J. Nucl. Energy 20: 411 (1966). One measurement at $22.8 \mathrm{keV}$ as corrected by Miller et al. in NucZ. Sci. Eng. 35: 295 (1969).

14. Menlove, H. O., et al., Nucl. Sci. Eng. 33: 24 (1968). Nine measurements at $24-503 \mathrm{kev}$. Original data.

15. Moxon, M. C., Report $\Lambda$ ERE-R6074 (1969).

White source, time of flight; $0.50-100 \mathrm{keV}$. Results average in decimal intervals. Date revised in 1970 (ref. 20).

16. Fricke, M. P., et al., IAEA Conf., Helsinki (1970). White source, time of flight; $1-100 \mathrm{keV}$. Results average in decimal intervals, $100-752 \mathrm{keV}$ point results. Original data.

17. de Saussure, G., et al., Nucl. Sci. Eng. 51: 385 (1973). White source, time of flight; $0.100-100 \mathrm{keV}$. Results average over decimal intervals. Original data. 
18. Langner, I., et a1., Report KFK-880 (1968).

Evaluation.

19. Davey, W. G., Nucl. Sci. Eng. 39: 337 (1970).

Evaluation.

20. Sowerby, M. G., et a1., Report AERE-R7273 (1973).

Evaluation.

21. Ponitkin, Y. G., et al., Report IAEA-CN-26, Helsinki (1970).

Ten measurements at $24-145 \mathrm{keV}$. Original data.

22. Ponitkin, Y. G., et al., At. Energ. 33: 782 (1972).

Two measurements at 132-160 keV: Original data.

23. Block, R. C., et al., Conf-720901, vol. II, p. 1107 (1972).

One measurement at $24 \mathrm{keV}$. Original data.

24. Chelnokov, V. B., et al., "Neutron cross section for radioactive capture," preprint USSR Institute of Physics and Power Engineering (1973).

Ten measurements at 3.3-35 keV. Original data.

25. Ryves, T. B., et a1., J. Nucl. Energy 27: 519 (1973).

Four measurements at 157-624 keV. Original data.

26. Pearlstein, S., et al., UKAEA Progress Report (1973).

Eleven measurements at 200-1750 keV. Original data.

27. Spencer, R. R., et al., private communication, 1975.

The following are ratio measurements relative to the ${ }^{235} \mathrm{U}$ fission cross section. They were reduced to ${ }^{238} \mathrm{~V}$ capture data utilizing the ${ }^{235} \mathrm{U}$ fission cross-section ENDF/B-IV evaluation. 
28. Linemberg, G. A., et a1., Report LA-467 (1946).

Thirteen measurements at $0.005-5.9 \mathrm{MeV}$. Original data.

29. Barry,.J. F., et al., J. Nucl. Energy 18: 481 (1964).

Thirteen measurements at $0.127-7.6 \mathrm{MeV}$. Original data.

30. Weston; L. W.; et al., Report EANDC 330, p. 64 (1963).

See also ref. 10 .

Two measurements at $30-64 \mathrm{keV}$. Original data.

31. Poenitz, W. P., Nucl. Sci. Eng. 49: 383 (1970) and Trans. Am: Nucl. Soc. 12: 279 (1968).

Thịteen, measurements at $30 \mathrm{keV}-1.4 \mathrm{MeV}$. Original data.

32. Sowerby, M. G., et al., Report AERE-R7273 (1973).

Evaluation.

$239 \mathrm{Pu}$ Capture: In this case the covariance $[\operatorname{COV}(\mathrm{I}, \mathrm{J})]$ was calculated for 36 groups for energies ranging from $1 \mathrm{MeV}$ to $100 \mathrm{eV}$. The energy range was divided in decimal intervals. The following set of references was used:

1. Sowerly, M. G., el al., Al. Erlergy Rev. 10(4): 453 (1972). IAEA.

2. Gwin, R., et a1., Nucl. Sci. Eng. 45:25 (1971).

3. Schomberg, M. G., et a1., p. 315 in Proc. Conf. Nuclear Data for Reactors, vo1. I, IAEA, Helsinki, 1970.

4. Czirr, J. R., et al., p. 331 in Proc. Conf. Nuclear Data for Reactors, vol: I, IAEA, Helsinki, 1970. 
5. Belyaer, F. N., et al., p. 336 in Proc. Conf. Nuclear Data for Reactors, vol. I, IAEA, Helsinki, 1970.

6. Farre1l, J. A., et al., p. 543 in Proc. Conf. Nuclear Data for Reactors, vol. I, IAEA, Helsinki, 1970.

7. Kononov, V. N., et al., p. 345 in Proc. Conf. Nuclear Data for Reactors, vol. I, IAEA, Helsinki, 1970.

8. Ryabov, Yu. V., et a1., p. 345 in Proc. Conf. Nuclear Data for Reactors, vo1. I, IAEA, Helsinki, 1970.

9. Weston, L. W., et al., memorandum to R. E. Chrien, 1972.

10. Gwin, R., et al., to be published in Nuclear Science Engineering.

$2{ }^{29} \mathrm{Pu}$ Fission: In this case the covariance $[\operatorname{COV}(I, J)]$ was calculated for 45 groups for energies ranging from $10 \mathrm{MeV}$ to $100 \mathrm{eV}$. The energy range was divided in decimal intervals. The following set of references was used:

1. Sowerby, M. G., et al., Report AERE- $\bar{R}-7273$, UKAEA (1973).

2. Hunter, R. E., et a1., Report LA-5172 (1973).

3. Gwin, R,, to be published in Nuclear Soience Engineering.

4. Weston, L. W., memorandum to R. E. Chrien, 1972.

5. James, G. D., p. 267 in Proc. Conf. Nuclear Data for Reactors, vo1. I, IAEA, Helsinki, 1970.

6. Gwin, R., et al., Nucl. Sci. Eng. 45: 25 (1971).

7. Shunk, E. R., et al., p. 979 in Proc. Conf. Neutron Cross Section Technology, vol. II, CONF-660303, 1966.

8. Blons, J., Nucl. Sci. Eng. 51: 95 (1973). 
9. Schomberg, M. G., et a1., p. 315 in Proc. Conf. Nuclear Data for. Reactors, vol. I, IAEA, Helsinki, 1970.

$240 \mathrm{Pu}$ and ${ }^{241} \mathrm{Pu}$ Capture. ${ }^{241} \mathrm{Pu}$ Fission: In this case the covariance was calculated for 45 groups for energies ranging from. $10 \mathrm{MeV}$ to. $100 \mathrm{eV}$. The following sets of references were used:

\section{Pu Capture Cross Section}

1. Weston, L. W., and Todd, J. H., "Measurement of the Neutron Capture Cross Sections of the Actinides," Proceedings of the Conference on Nuclear Cross Sections and Technology, Washington, D.C., 1975, to be published.

$100 \mathrm{eV}$ to $300 \mathrm{keV}$ with weight of 9 on the variance.

2. Hockenbury, R. W., Moyer, W. R.; and Block, R.'C., Nucl. Sci. Eng. 49: $153-161$ (1972).

6 to $30 \mathrm{keV}$ with weight of 9 on the variance.

3. Prince, A., Conf. Nucl. Data for Reactors, CN-26/91, Helsinki, 1970.

$1 \mathrm{keV}$ to $10 \mathrm{MeV}$ with, weight of 1 on the variance (model calculation).

4. Yiftah, S., Schmidt, J. J., Caner, M., and Segev, M., p. 123 in Fast Reactor Physics, vol. I, IAEA, Vienna, 1968.

$1 \mathrm{keV}$ to $10 \mathrm{MeV}$ with weight of 1 on the variance (model calculation). 


\section{$241 \mathrm{Pu}$ Capture Cross Section}

1. Weston, L. W., and Todd, J. H., "Neutron Fission and Absorption Cross Sections for ${ }^{239} \mathrm{Pu}$ and $241 \mathrm{Pu}, "$ Trans. Am. Nucl. Soc. 15(1): 480 (1972) $100 \mathrm{eV}$ to $200 \mathrm{keV}$ with weight of 9 on the variance.

2. Caner, M., and Yiftah, S., p. 735 in Conf. Nucl. Data for Reactors, vol. II, IAEA, Vienna, 1970.

$100 \mathrm{eV}$ to $10 \mathrm{MeV}$ with weight of 1 on the variance (model calculation)

3. Prince, A., Conf. Nucl. Data for Reactors, CN-26/91, Helsinki, 1970. $80 \mathrm{keV}$ to $10 \mathrm{MeV}$ with weight of 1 on the variance (model calculation).

$24{ }^{\mathrm{Pu}}$ Fission Cross Section (all references with weight of 1 on the variance)

1. Weston, L. W., and Todd, J. H., same reference as with ${ }^{241} \mathrm{Pu}$ capture: $100 \mathrm{eV}$ to $200 \mathrm{keV}$.

2. Simpson, 0. D., et a1., p. 910 in Proc. Conf. on Neutron Cross Section and Technology, vol. II, Washington, D.C., 1966. $100 \mathrm{eV}$ to $10 \mathrm{keV}$.

3. Blons, J., et al., p. 469 in Conf. Nucl. Data for Reactors, vo1. I, Vienna, 1970.

4. Migneco, E., Theohald, J. P., and Wartena, J. A., p. 437 in Conf. Nuclear Data for Reactors, vol. I, Vienna, 1970. $100 \mathrm{eV}$ to $2 \mathrm{keV}$. 
5. Smith, H. L., Smith, R. K., and Henkel, R. L., Phys. Rev. 125: 1329 (1962).

$200 \mathrm{keV}$ to $10 \mathrm{MeV}$.

6. Zabo, I., et al., Neutron Standards and Flux Normalization, p. 256, Argonne National Laboratory (1970).

$20 \mathrm{keV}$ to $1 \mathrm{MeV}$.

7. White, P. H., and Warner, G. P., J. Nucl. Energy 21: 671 (1967). $40 \mathrm{keV}$ to $6 \mathrm{MeV}$.

8. Butler, D. K., and Sjoblom, R. L., Phys. Rev. 124: 1129 (1961). $300 \mathrm{keV}$ to $2 \mathrm{MeV}$. 
THIS PAGE

WAS INTENTIONALLY

\section{LEFT BLANK}


APPENDIX B. STANDARD DEVIATION AND CORRELATION MATRICES CORRESPONDING TO DIFFERENT CROSS SECTIONS 


\section{U238 FISSION 36 GROUPS}

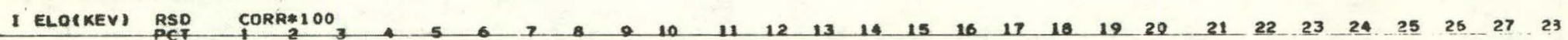

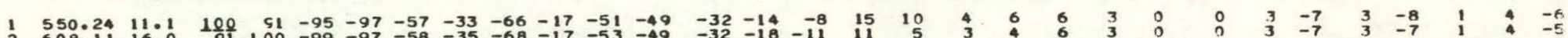

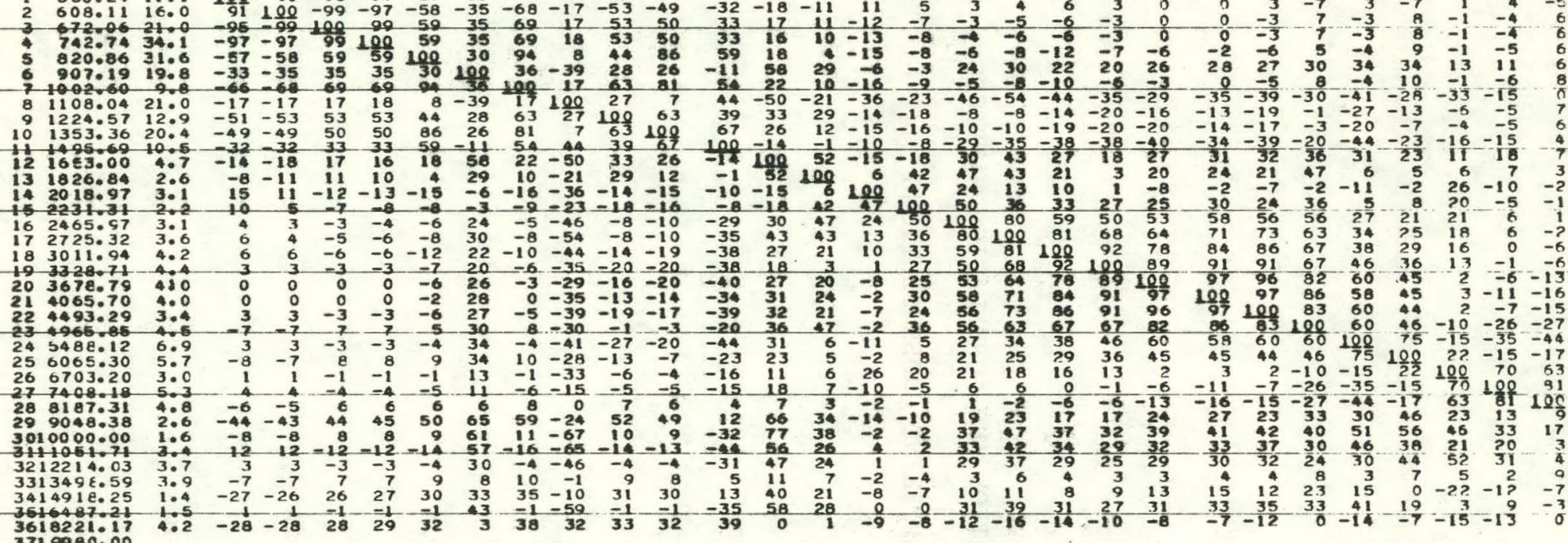

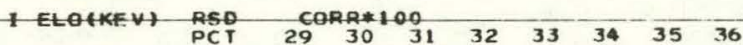

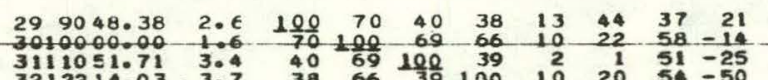

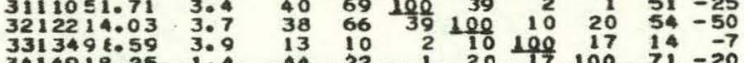

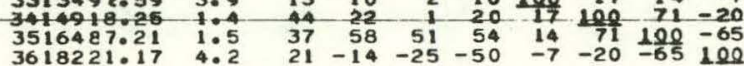

3618221.17 
U23B CAP TURE 91 GROUPS

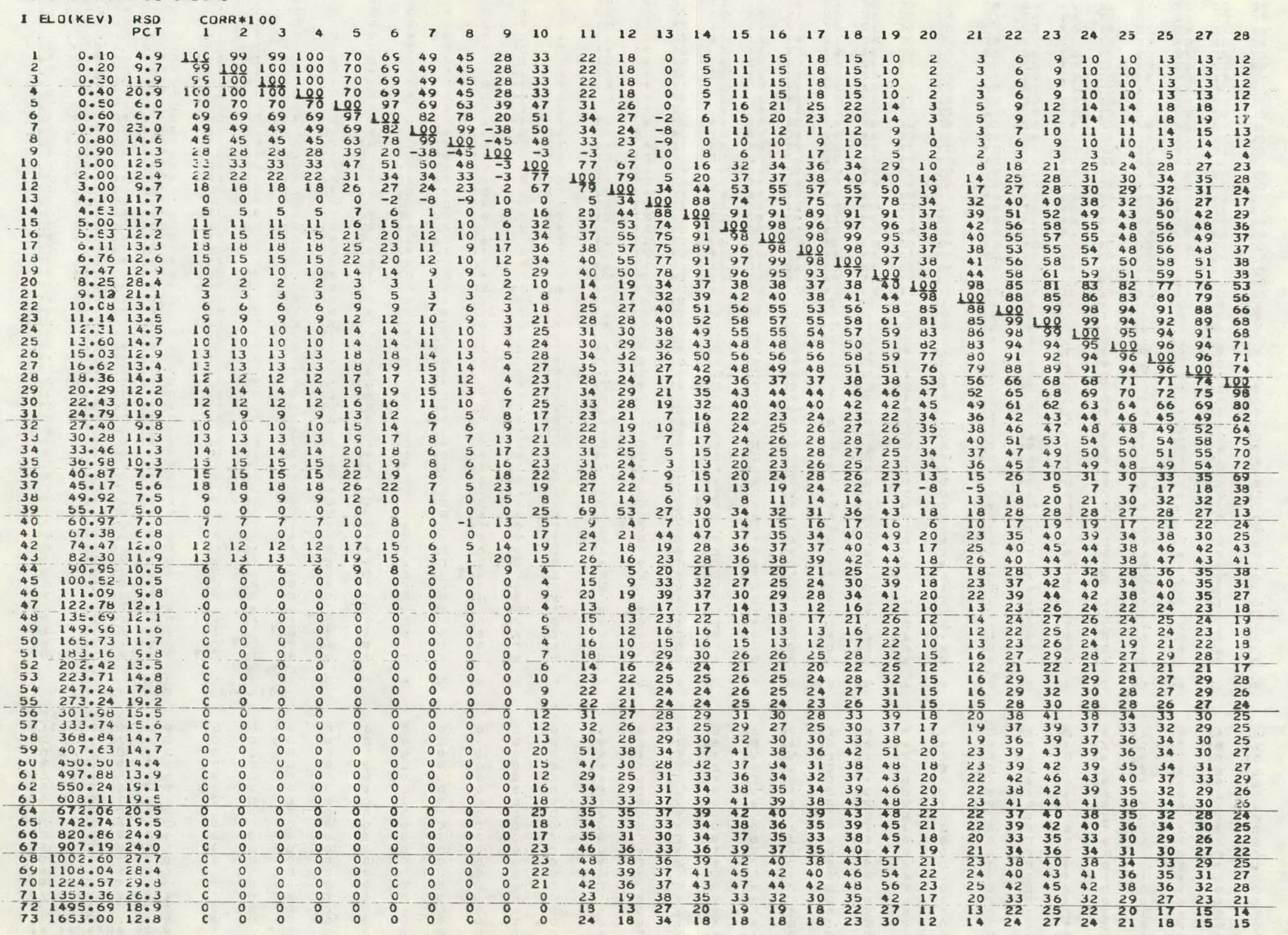




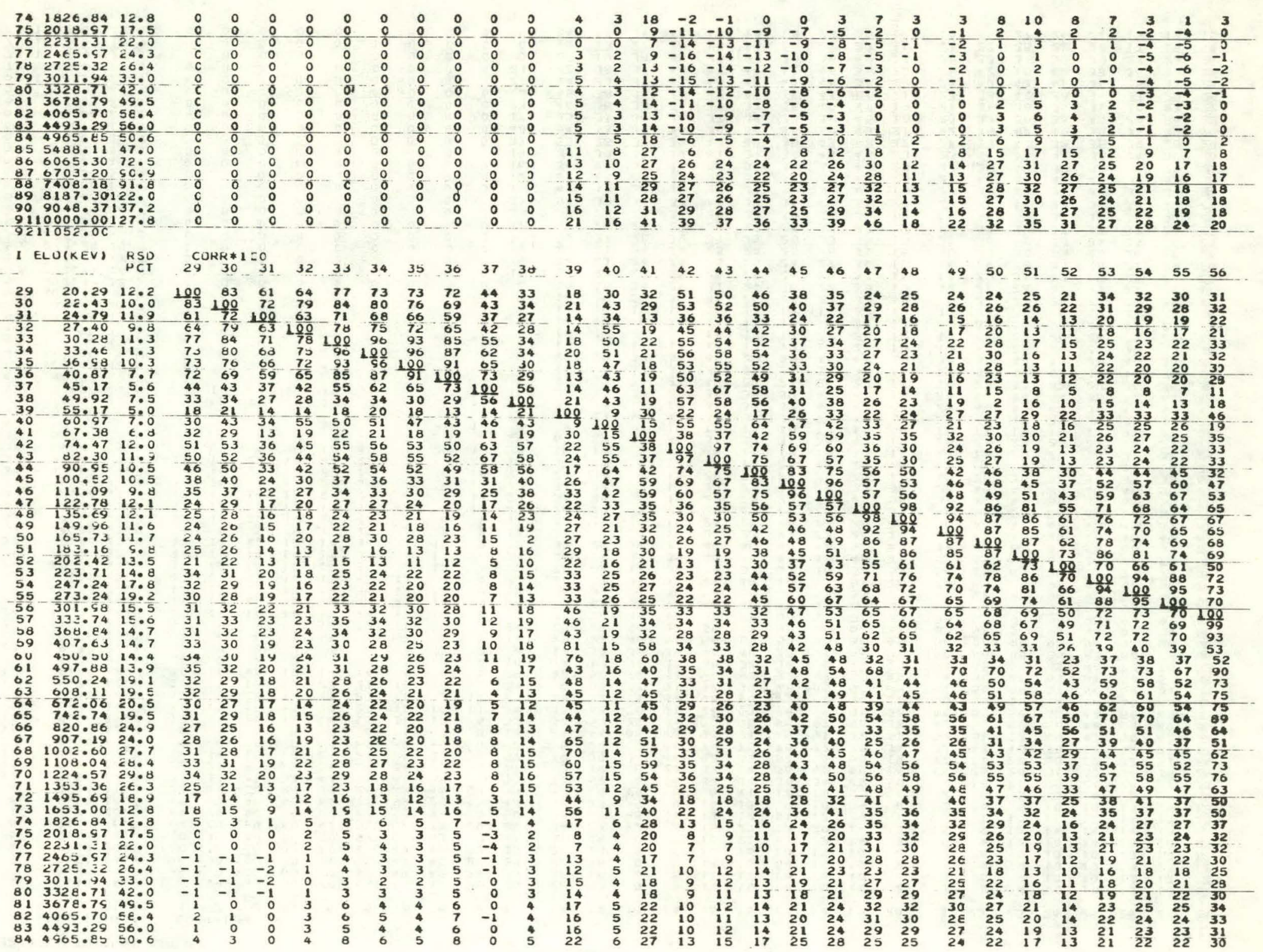




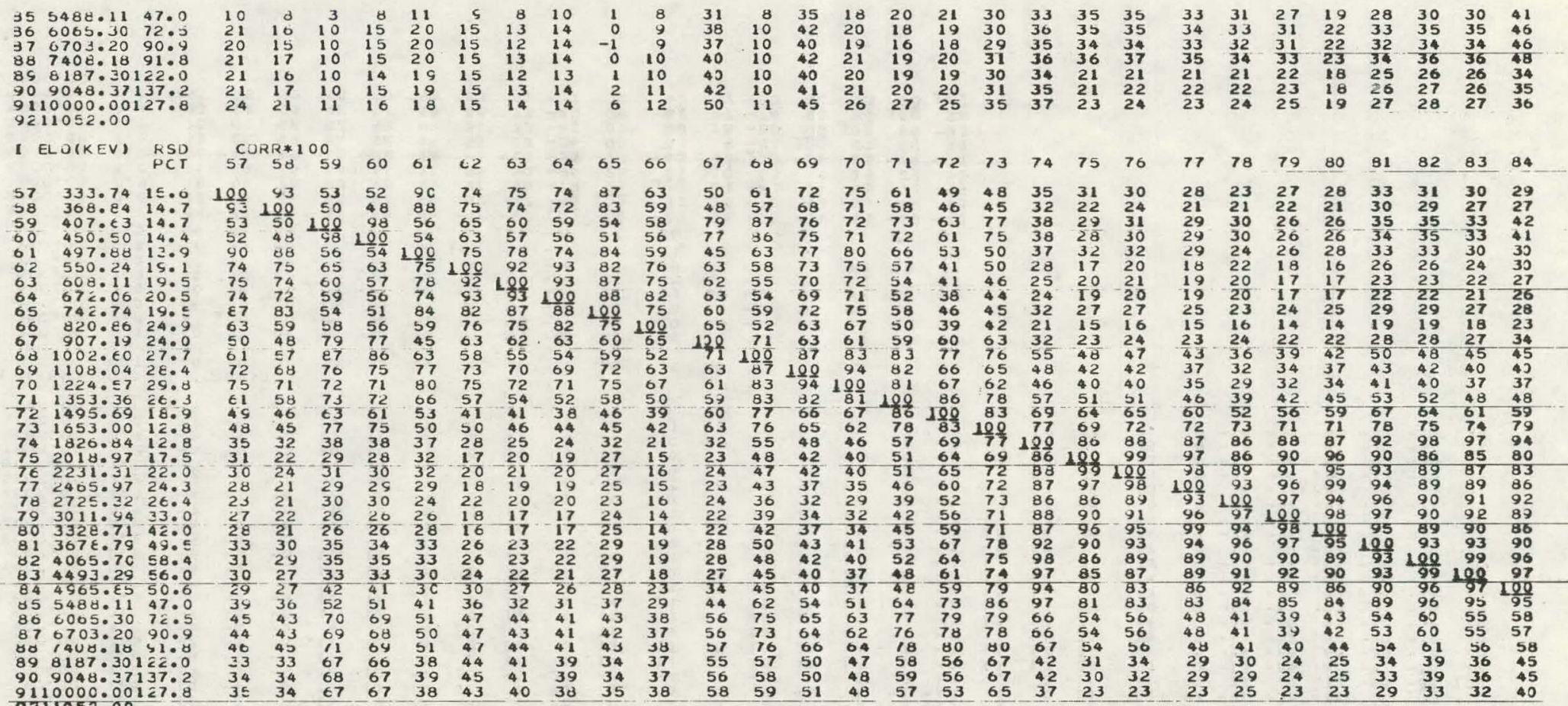

\begin{tabular}{lllllll}
1 & ELOUKEV) RSO ROP CORR*100 \\
\hline
\end{tabular}

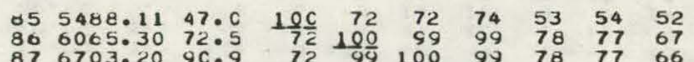

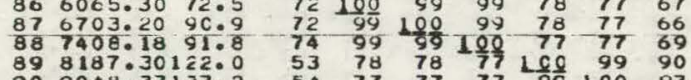

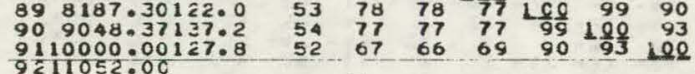


PU 239 FISSION 45 GROUPS

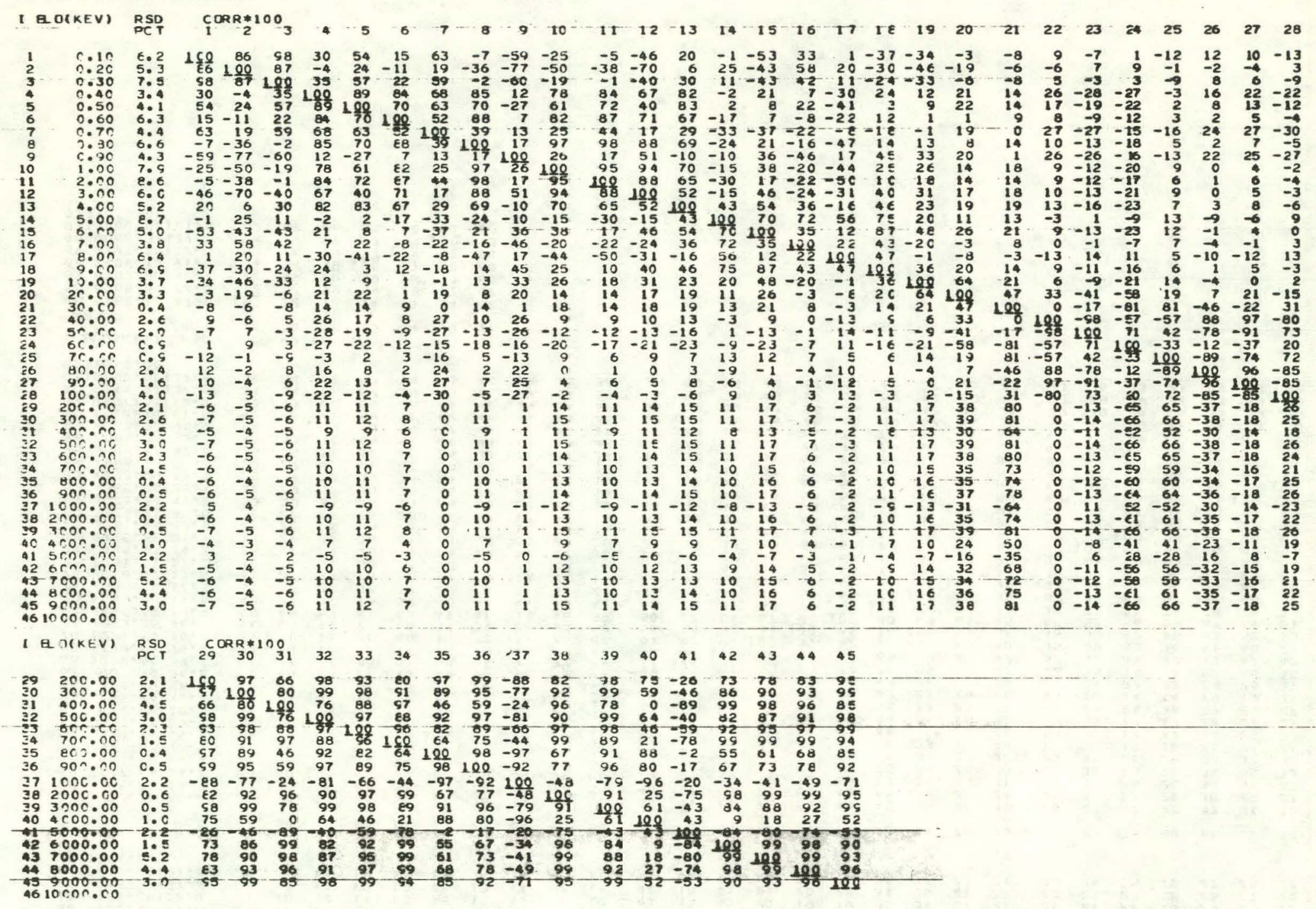


PU 239 CAPTURE 36 GLOUPS

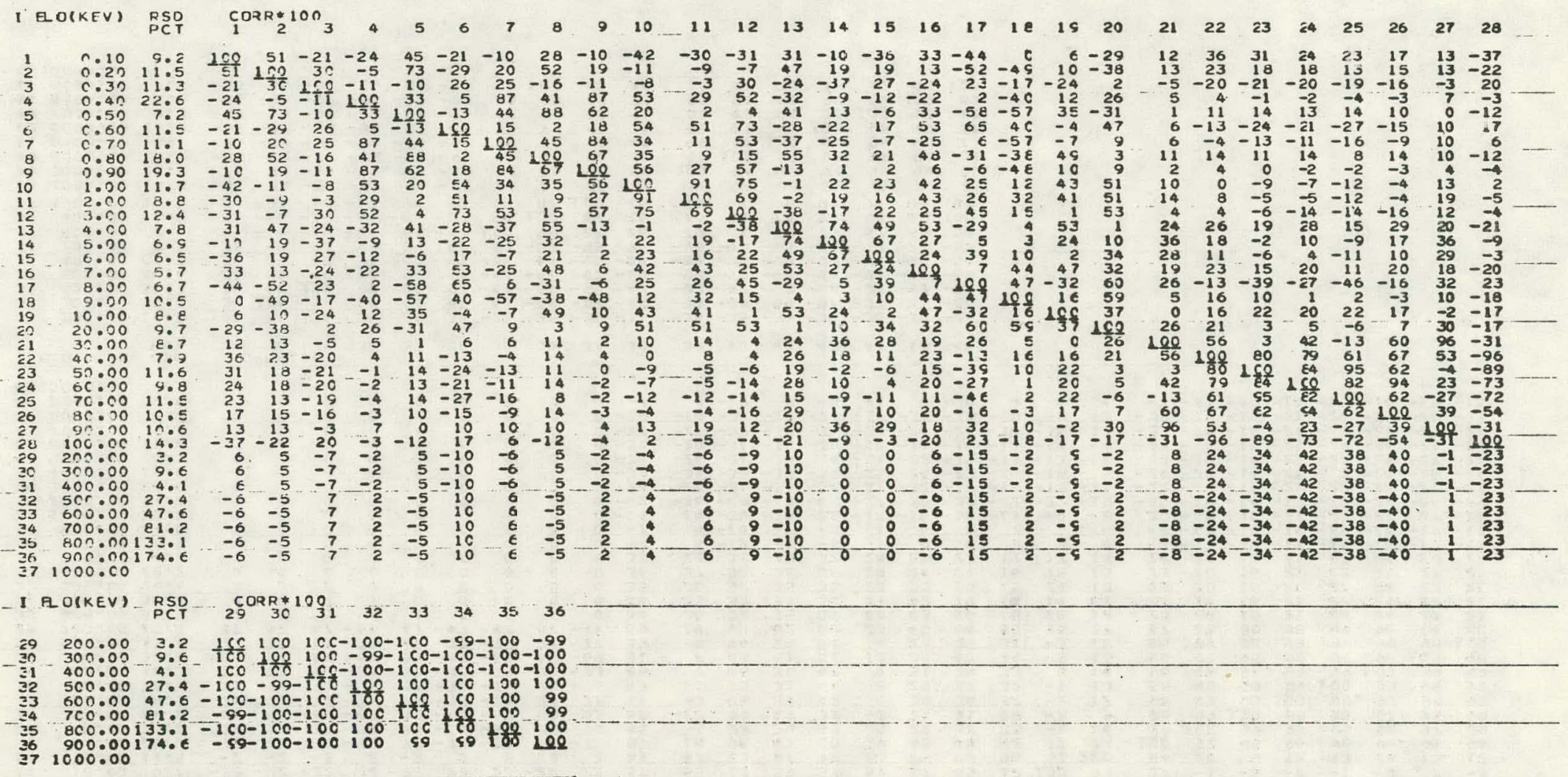




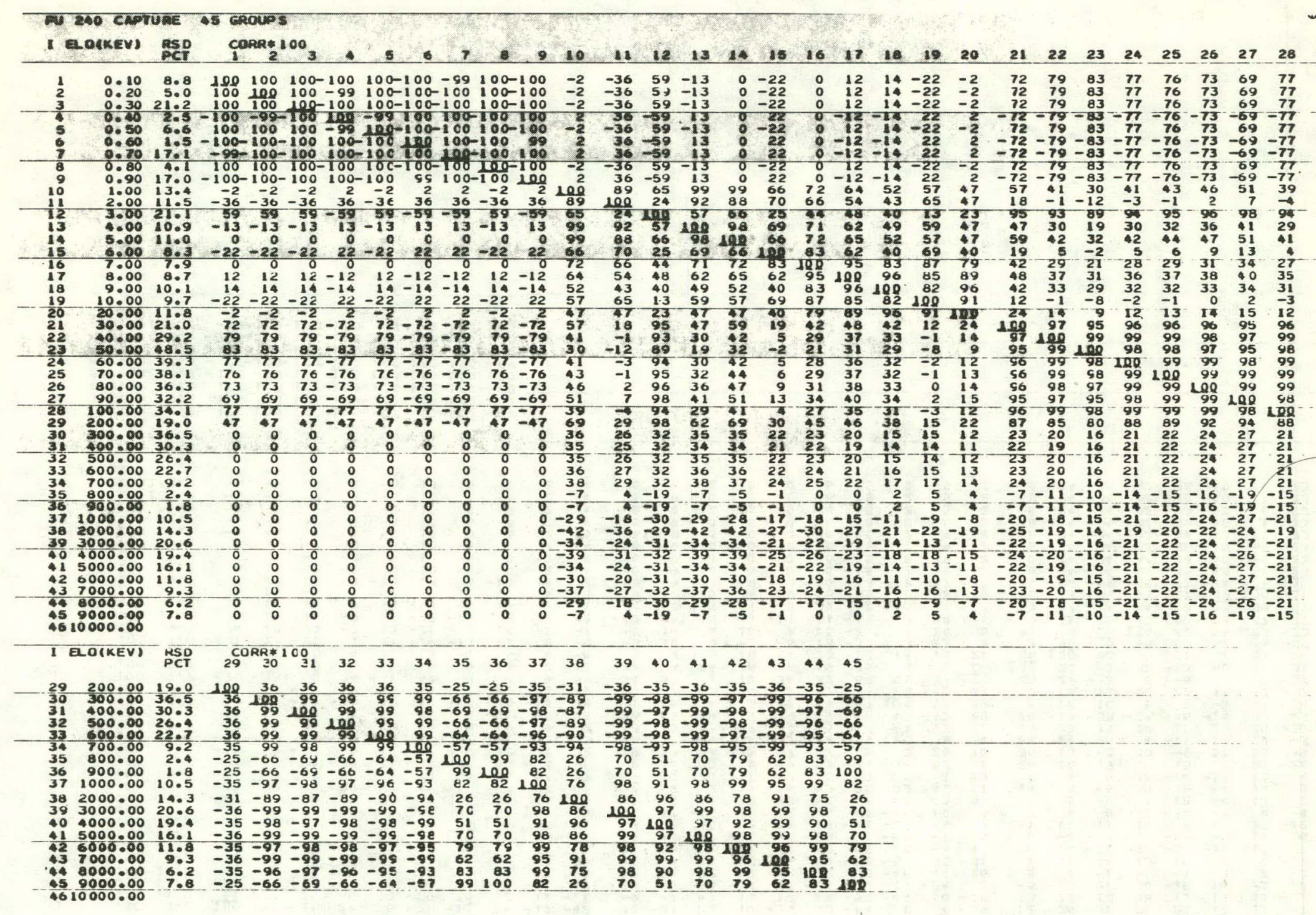




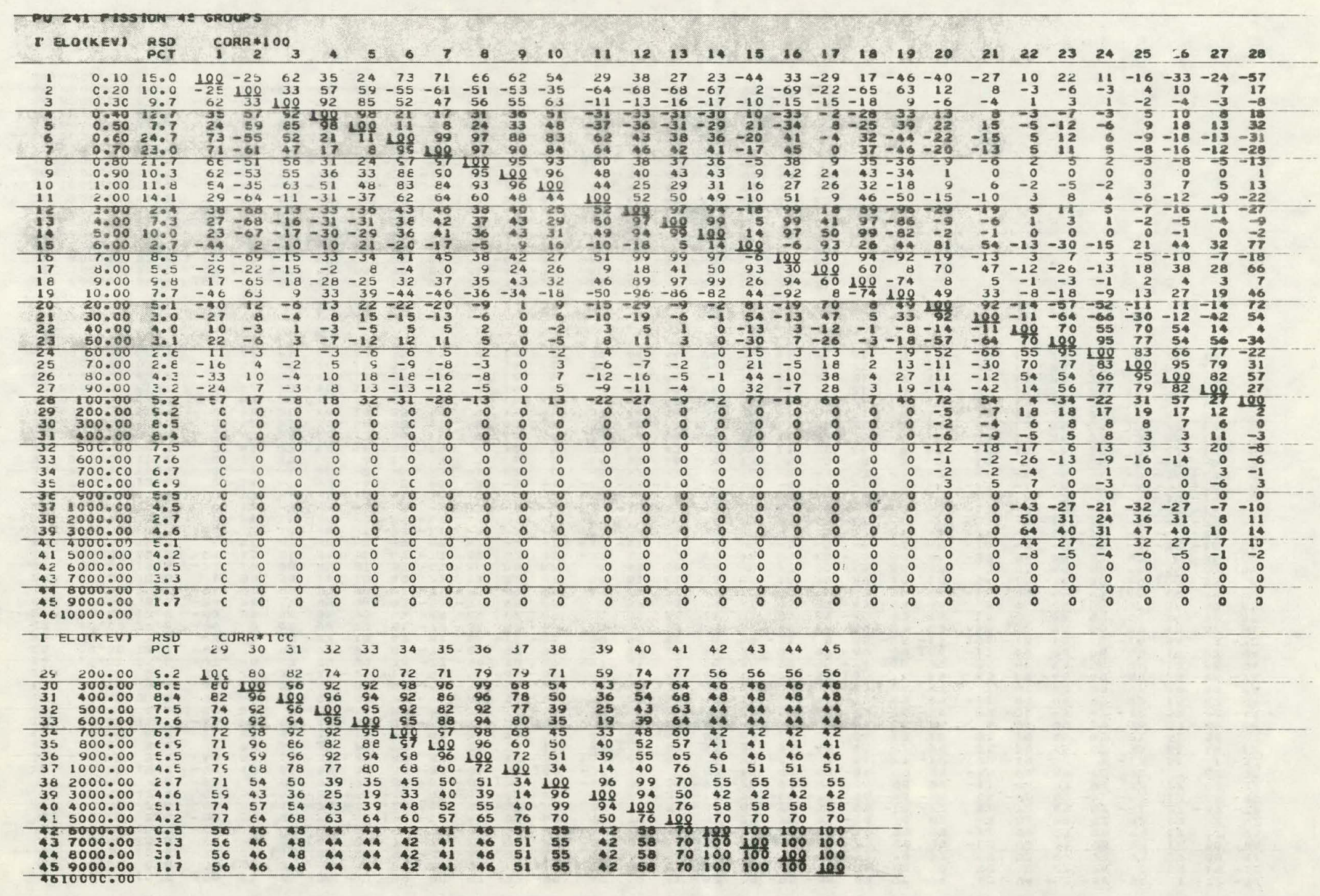




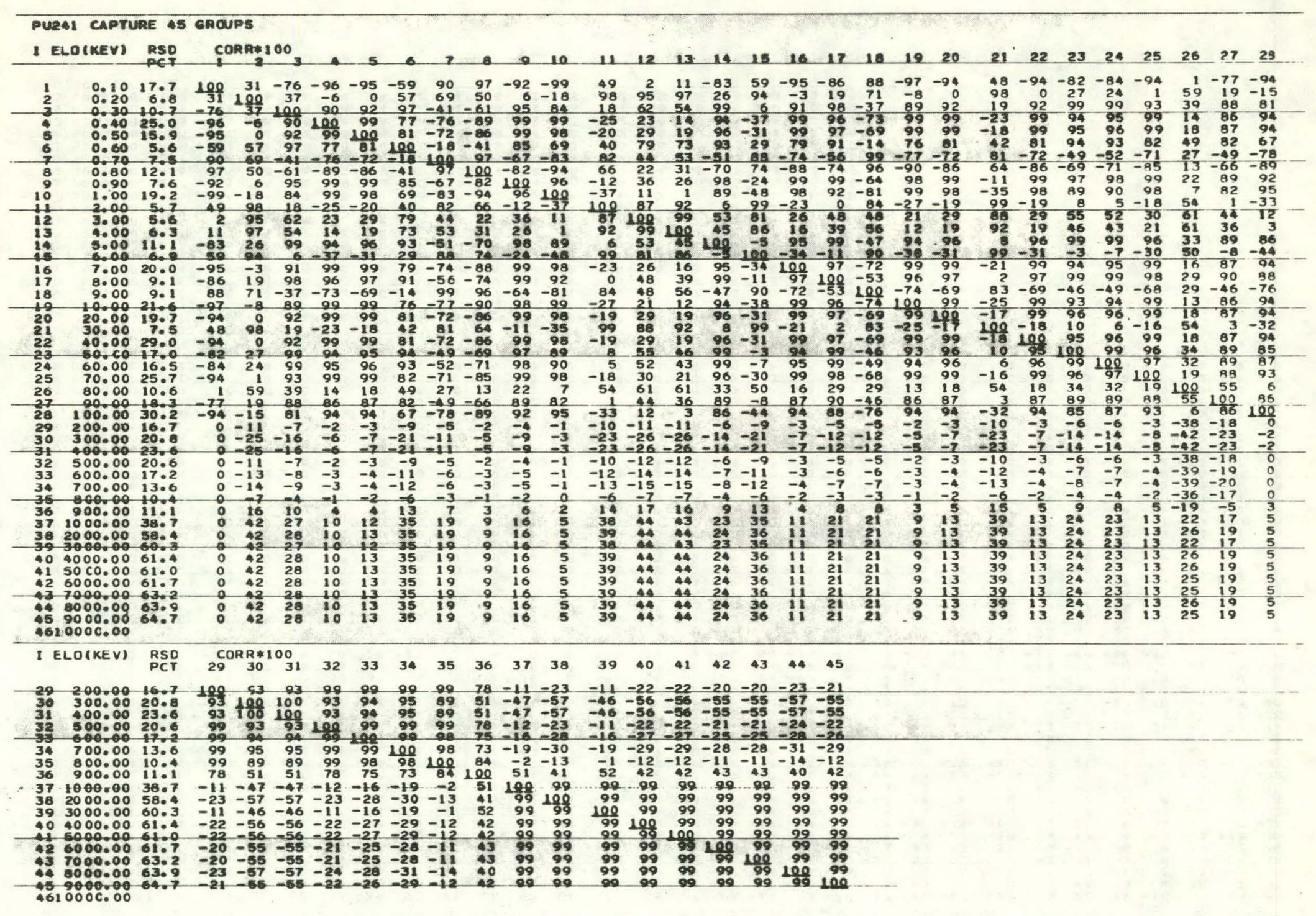


INTERNAL DISTRIBUTION

ORNL/TM-5223

UC-79d

1-3 L. S. Abbott

4 G. de Saussure

5-11 F. C. Difilippo

12 J. D. Drischler

13 G. F. Flanagan

14 C. Y. Fu

15 H. Goldstein (consultant)

16 R. Gwin

17 D. C. Larson

18 J. H. Marable

19 F. R. Mynatt

20 D. K. Olsen

21-50 R. W. Peelle

51 F. G. Perey

52-61 R. B. Perez
62 M. L. Tobias

63-67 C. R. Weisbin

68 L. W. Weston

69 A. Zucker

70 P. F. Fox (consultant)

71 W. W. Havens, Jr. (consultant)

72 A. F. Henry (consultant)

73 R. E. Uhrig (consultant)

74-75 Central Research Library

76 ORNL Y-12 Technical Library, Document Reference Section

77-78 Laboratory Records Department

79 Laboratory Records Dept. ORNL RC

80 ORNL Patent office

EXTERNAL DISTRIBUTION

81 USERDA Oak Ridge Operations, Research and Technical Support Division, P. 0. Box E, Oak Ridge, Tennessee 37830: Director

82 USERDA Oak Ridge Operations, Reactor Division, P. O. Box E, Oak Ridge, Tennessee 37830: Director.

83-84 USERDA Division of Reactor Research and Development, Washington, D. C. 20545: Director.

85-290 For distribution as shown in TID-4500 Distribution Category, UC-79d, Liquid Meta1 Fast Breeder Reactors (Physics). 